\title{
st \\ Aritmetizando la geometría desde dentro: el cálculo de segmentos de David Hilbert
}

\author{
Eduardo Nicolás Giovannin I
}

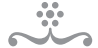

RESUMEN

Sobre la base que aportan las notas manuscritas de David Hilbert para cursos sobre geometría, el artículo procura contextualizar y analizar una de las contribuciones más importantes y novedosas de su célebre monografía Fundamentos de la geometría (1899), a saber: el cálculo de segmentos lineales (Streckenrechnungen). Se argumenta que, además de ser un resultado matemático importante, Hilbert depositó en su aritmética de segmentos un destacado significado epistemológico y metodológico. En particular, se afirma que para Hilbert este resultado representaba un claro ejemplo de uno de los rasgos más fructíferos y atractivos de su nuevo método axiomático formal, o sea, la capacidad de descubrir y exhibir conexiones estructurales o internas entre diferentes teorías matemáticas.
\end{abstract}

PALABRAS-CLAVE • Hilbert. Método axiomático. Unidad de la matemática. Geometría euclídea.

Aritmetización.

\section{INTRODUCGIÓN}

El 17 de junio de 1899 David Hilbert (1862-1943) publicó un breve trabajo dedicado a los fundamentos de la geometría, que formó parte de un volumen celebratorio (Festschrift) en ocasión de la inauguración en Göttingen de un monumento en homenaje a Carl Friedrich Gauss (1777-1855) y Wilhem Weber (1804-1891). La monografía llevó el nombre Fundamentos de la geometría (Hilbert, 1899) y es considerada actualmente no sólo como una de sus contribuciones más importantes a los fundamentos de la matemática, sino también como un hito fundamental de la concepción moderna o abstracta del método axiomático. Hilbert presentó allí el primer sistema axiomático formal para la geometría euclídea elemental, a través del cual era posible desarrollar esa teoría matemática de un modo riguroso y sistemático. Su exposición capturó la marcada tendencia hacia la abstracción y sistematización experimentada por la matemática en la segunda mitad del siglo xIx, convirtiendo definitivamente a la geometría en una teoría matemática pura. Dicho trabajo tuvo además el enorme mérito de haber inaugurado una nueva y fructífera área de investigación matemática, a saber: la mate- 
mática de los axiomas o metamatemática. En efecto, Hilbert llevó a cabo una investigación sistemática de la consistencia, completitud y, principalmente, de la independencia de un axioma o conjunto de axiomas, y arribó a numerosos resultados "metageométricos" que contribuyeron a esclarecer el lugar de diversos teoremas fundamentales como por ejemplo los teoremas de Desargues y Pascal - en la estructura de la geometría euclídea elemental.

Ahora bien, otra contribución importante presentada en Fundamentos de la geometría consistió en la construcción de distintos cálculos de segmentos lineales (Streckenrechnungen). Hilbert mostró cómo era posible definir las operaciones de suma y multiplicación de segmentos lineales de un modo puramente geométrico y al mismo tiempo probó, recurriendo a los teoremas clásicos de Desargues y de Pascal, que esas operaciones satisfacían todas las propiedades de un cuerpo ordenado. Esa construcción puramente geométrica de un conjunto que satisface la estructura de un cuerpo ordenado le permitió reconstruir la clásica teoría euclídea de las proporciones y de los triángulos semejantes, la cual finalmente le sirvió para llevar a cabo una aritmetización interna o "desde dentro" de la geometría.

La importancia de la aritmética de segmentos presentada por Hilbert en Fundamentos de la geometría fue reconocida inmediatamente y ha sido mencionada a menudo como una contribución importante a los fundamentos de la geometría. ${ }^{1}$ Sin embargo, en este trabajo sostendré que ése no sólo fue un resultado matemático destacado, sino que además Hilbert le confirió una gran relevancia metodológica y epistemológica. En particular, argumentaré que para Hilbert su aritmética de segmentos ponía de manifiesto uno de los rasgos o características más novedosos y atractivos de su nuevo método axiomático formal, desde un punto de vista matemático. Este rasgo consistía en la capacidad del método axiomático de descubrir y exhibir conexiones internas o estructurales entre teorías matemáticas de muy diversa índole y así contribuir a la unidad del conocimiento matemático. En este sentido, Hilbert enfatizó que el método axiomático no sólo debía ser concebido como un instrumento eficaz para presentar una teoría matemática de un modo más perspicuo y lógicamente preciso, sino además - y aun no menos importante - como una herramienta sumamente fecunda para el descubrimiento de nuevos resultados matemáticos.

1 La importancia de la aritmética de segmentos de Hilbert es mencionada en los artículos clásicos de Blumenthal (1935) y Freudenthal (1957). En cuanto a la recepción inmediata, Hessenberg (1905b) y Hölder (1911) construyeron nuevos cálculos de puntos y segmentos basándose en las ideas originales de Hilbert. Desde un punto de vista más filosófico, la relevancia de ese resultado ha sido destacada por Webb (1980) y Rowe (2000). Finalmente, Hartshorne (2000) resalta la importancia de los resultados alcanzados por Hilbert y los presenta de acuerdo con una forma más contemporánea. 
Para defender mi tesis me serviré extensamente de un conjunto de manuscritos, consistentes en notas de clases cuidadosamente elaboradas para cursos sobre geometría dictados por Hilbert entre 1891 y 1902. Tales notas de clases han sido publicadas parcialmente en Majer y Hallett (2004), mientras que las fuentes originales se hallan depositadas en la Niedersächsische Staats und Universitätsbibliothek Göttingen, Handschriftenabteilung, y en la Bibliothek des mathematischen Seminars, Lesesaal, Georg-AugustUniversität Göttingen. ${ }^{2}$ La importancia de esas fuentes reside en que Hilbert no sólo desarrolló allí sus investigaciones geométricas y, especialmente metageométricas, de un modo más detallado, sino que además los resultados alcanzados estuvieron acompañados por numerosas reflexiones y observaciones respecto de su significado metodológico y epistemológico. En consecuencia, las notas de clases resultan un material invaluable para apreciar y comprender el contexto y el significado que Hilbert le confirió a sus primeras investigaciones axiomáticas en el ámbito de la geometría.

El artículo se organiza de la siguiente manera. En la primera sección utilizaré los manuscritos de Hilbert para mostrar cómo el problema de la introducción del número en geometría constituyó desde bien temprano un problema muy importante en su nuevo abordaje axiomático a la geometría. En la segunda sección, señalaré cómo Hilbert consideró que el método axiomático formal resultaba particularmente útil para analizar este problema. En la tercera sección me ocuparé de exponer los resultados fundamentales de su cálculo de segmentos, según es presentado en Fundamentos de la geometría. Finalmente, en la última sección concluiré que Hilbert concibió ese resultado como un claro ejemplo de la "unidad orgánica de la matemática", una unidad que ahora podía ser investigada de un modo sistemático gracias a su nueva concepción abstracta del método axiomático.

\section{Antegedentes en la geOmetría del XIX}

\subsection{HiLbert Y LA TRADición DE LA GEOMETRÍA SINTÉTICA}

Uno de los aspectos más interesantes de las notas de clases de Hilbert consiste en la posibilidad de identificar las fuentes y problemas fundamentales que motivaron sus investigaciones axiomáticas en el campo de la geometría. ${ }^{3}$ En particular, estos cursos muestran claramente que Hilbert inscribió su propio abordaje axiomático dentro de la tradición de la geometría sintética. Como es bien sabido, los métodos geométricos “pu-

2 Para una idea general de la naturaleza de esas notas manuscritas de clases, véase Majer y Hallett (2004).

3 Esta tarea fue emprendida por primera vez en el trabajo pionero de Toepell (1986). Véase además Corry (2004). 
ros" recobraron un renovado interés hacia fines del siglo xviII, a partir del surgimiento de la geometría proyectiva como una nueva teoría matemática. 4 'Entre los representantes más importantes de esta tradición se encuentran Gaspard Monge (1746-1818), Lazare Carnot (1785-1823) y Victor Poncelet (1788-1867) en Francia, y posteriormente Jakob Steiner (1796-1863) y Christian von Staudt (1798-1867) en Alemania. Esencial para esta tradición resultaba prescindir de la introducción de consideraciones numéricas en la construcción de la geometría, esto es, evitar la formulación algebraica de relaciones geométricas que desde la aparición de La géométrie de Descartes en 1637 había probado ser tan exitosa. En cambio, hacia comienzos del siglo xix surgió gradualmente un programa de investigación que intentaba descubrir y desarrollar técnicas puramente geométricas para las investigaciones de diversos objetos geométricos en el plano y en el espacio. Un ejemplo de este nuevo tipo de investigaciones geométricas consistió en la utilización que hizo Poncelet de la correspondencia polar entre puntos y líneas relativos a una sección cónica para traducir teoremas acerca de la configuración de puntos en teoremas acerca de la configuración de líneas, y viceversa. Este programa de investigación fue continuado en Alemania por Steiner y von Staudt, a quienes se les deben quizás las presentaciones más sistemáticas y acabadas de la geometría proyectiva desde un punto de vista sintético (cf. Gray, 2007; Nabonnand, 2008b).

Por otra parte, algunos geómetras franceses y alemanes notaron inmediatamente que las propiedades proyectivas que habían comenzado a ser el centro de las nuevas investigaciones geométricas podían ser igualmente tratadas por medio de ecuaciones algebraicas. En particular, August Möbius (1790-1868) y Julius Plücker (1801-1868) fueron los primeros en introducir coordenadas homogéneas en la geometría proyectiva, con lo cual se volvió patente que la nueva geometría podía ser formulada e investigada tanto sintética como analíticamente. ${ }^{5}$ De este modo, un objetivo explícito de los geómetras sintéticos consistió en intentar lograr una presentación de la geometría proyectiva con un grado de generalidad, abstracción y rigor equiparable al de la geometría analítica, pero sin renunciar a su carácter puramente geométrico, es decir, sin impulsar una reducción de la geometría al álgebra por medio de la utilización del método de las coordenadas. ${ }^{6}$

4. Sobre el renacimiento de los métodos geométricos puros hacia fines del siglo xviı, y comienzos del siglo XIX, véase Kline (1992, cap. 39).

5 Sobre las discusiones metodológicas entre geómetras analíticos y sintéticos en el siglo xIx, en el seno de la geometría proyectiva, véase Epple (1997).

6 Sobre la búsqueda de generalidad en las demostraciones dentro de la tradición sintética puede verse Nabonnand (2011). Por otro lado, una fuente fundamental para la comprensión de este proceso sigue siendo el clásico artículo de Nagel (1939). 
La cuestión acerca de si la nueva geometría proyectiva debía ser abordada utilizando técnicas geométricas puras o métodos algebraicos se convirtió hacia la mitad del siglo XIX en una acalorada controversia. Sin embargo, como lo observó Felix Klein (1849-1925), las discusiones se plantearon fundamentalmente en relación al requisito metodológico de la "pureza del método". ${ }^{2}$ En efecto, para apreciar que la controversia estaba planteada en este nivel sólo basta observar el argumento central esgrimido por los geómetras sintéticos en contra de las técnicas analíticas. Para los geómetras sintéticos, los resultados alcanzados a través de métodos algebraicos difícilmente podían ser considerados como verdaderamente geométricos, en tanto era evidente que en la serie de manipulaciones algebraicas de las ecuaciones de las figuras geométricas resultaba imposible seguir cada uno de los pasos geométricos que correspondían a las operaciones algebraicas realizadas. El método analítico no sólo ocultaba el significado geométrico de los resultados alcanzados, sino que además por su intermedio llegábamos a afirmaciones sin saber realmente cuál era su lugar dentro del sistema de las verdades geométricas. Como se pregunta Michel Chasles (1793-1880), uno de los más férreos defensores de los métodos geométricos puros en Francia: “¿Es entonces suficiente en un estudio filosófico y básico de una ciencia saber que algo es verdadero si uno no sabe por qué es así y qué lugar debería ocupar en la serie de verdades a las que pertenece?" (Chasles apud Kline, 1992, p. 1104).

La preferencia de Hilbert por el abordaje sintético se aprecia tempranamente en un primer curso que dedica a la geometría proyectiva, dictado en el semestre de verano de $1891 .{ }^{\mathbf{8}}$ La organización y estructura de este curso sigue muy de cerca la tercera edición del libro Geometrie der Lage (1886) de Theodor Reye, el cual estaba basado a su vez en la presentación de la geometría proyectiva realizada por von Staudt (1847). En la introducción del curso, Hilbert presenta unas breves consideraciones históricas respecto del desarrollo de los métodos geométricos desde la antigüedad, y advierte lo siguiente respecto del método de las coordenadas introducido por Descartes:

7 "La geometría sintética es aquella que estudia las figuras en cuanto tales, sin recurrir a fórmulas, mientras que la geometría analítica utiliza consistentemente dichas fórmulas, a partir de la adopción de un sistema apropiado de coordenadas. Correctamente entendidos, solamente existe entre estos dos tipos de geometrías una diferencia de gradación, en tanto se le otorgue mayor importancia a las figuras o a las fórmulas. (...) En matemática, sin embargo, como en cualquier otro lugar, el hombre se inclina por formar partidos, de modo que así surgieron escuelas de [geómetras] 'sintéticos' puros y escuelas de [geómetras] 'analíticos' puros, quienes pusieron un énfasis primordial en la absoluta "pureza del método"” (Klein, 1949, p. 55).

8 Hilbert realizó prácticamente toda su formación académica, como así también sus primeros pasos como docente universitario, en la universidad de Königsberg, su ciudad natal. Su traslado a la universidad de Göttingen ocurrió en 1895, gracias a las gestiones de Felix Klein. Más información biográfica puede encontrarse en Reid (1996) y Rowe (2000). 
Este razonamiento [el método de las coordenadas] hace que de un golpe todo problema geométrico sea accesible al análisis [matemático]. Descartes se convirtió entonces en el creador de la geometría analítica. Inicialmente los teoremas de los griegos fueron de nuevo demostrados y luego generalizados. En lugar del ingenio [Kunstgriffe] aparecieron las fórmulas, el cálculo - y gracias a Descartes, un método real. Y así como estos avances fueron tan importantes y tan magnífico fue su éxito, así también sufrió finalmente la geometría bajo la educación unilateralmente orientada de este método. Ahora sólo se calculaba, sin tener la intuición de lo calculado. Se perdió el sentido por la figura y la construcción geométrica (Hilbert, 2004 [1891], p. 24). 9

Hilbert considera el resurgimiento de los métodos geométricos puros en los trabajos de Monge, Poncelet, Chasles y von Staudt como una reacción ante la pretendida reducción de la geometría al álgebra y el análisis, sugerida por los geómetras analíticos. Más aún, la cuestión de la "pureza del método" es mencionada explícitamente en relación a los trabajos de von Staudt (1847), a quien elogia por haber conseguido presentar a la geometría proyectiva como una ciencia autónoma, independiente de consideraciones métricas:

Contrariamente a todos sus antecesores, quienes siempre necesitaron del cálculo, él [von Staudt] consiguió hacer de la geometría proyectiva "un ciencia autónoma, que no requiere de la medida" - como él mismo lo afirma en el prólogo. Él [von Staudt] logró una geometría en la que no se calcula ni se mide, sino que se construye, en la que no se utiliza el compás ni el transportador, sino sólo la regla. De este modo aquel requerimiento científico fue cumplido de manera satisfactoria, puesto que en la deducción de los teoremas sobre las relaciones de posición, el cálculo debe aparecer como algo extraño. Presentada de esta forma, la geometría proyectiva constituye sólo una parte de la geometría, pero de hecho un dominio [dotado de] una unidad y conclusividad maravillosas. De acuerdo con el modelo presentado en esta obra he dado forma a mi curso sobre geometría proyectiva (Hilbert, 2004, [1891], p. 25)

9 Quizás sea oportuno realizar una breve observación acerca de la llamada "geometría analítica" de Descartes. A diferencia de lo que sugiere Hilbert en este pasaje, es preciso advertir que el método cartesiano no consistió meramente en establecer un simple y puro isomorfismo entre las líneas y curvas geométricas, por un lado, y las ecuaciones algebraicas, por otro lado. Por el contrario, la relación entre geometría y álgebra, con sus respectivos estatus epistemológicos, objetos, métodos y problemas, era para Descartes mucho más compleja y matizada. Para evitar esta simplificación habitual en la literatura no especializada puede consultarse, por ejemplo, Bos (1981). Agradezco a un árbitro de Scientiae Studia por llamar mi atención sobre este punto. 
Hilbert elogia de ese modo el "purismo metodológico" de von Staudt, que consiste no sólo en haber construido a la geometría proyectiva de una manera estrictamente sintética o pura, sin apelar al método de las coordenadas homogéneas introducido previamente por Möbius y Steiner, sino además en haber podido definir los conceptos y leyes fundamentales de esta teoría geométrica sin hacer recurso a ninguna consideración métrica. Más aún, en un breve pasaje de sus "Cuadernos científicos" (Wissenschaftliche Tagebücher), correspondiente a esta misma etapa, Hilbert repite este "requerimiento científico" que consiste en exigir que la geometría sea construida como una teoría autónoma o autosuficiente, independiente del álgebra y el análisis:

La geometría no va tan profundo como el análisis. Si uno se dedica a la geometría, entonces ésta debe ser sintética. [Pues], ¿Qué tiene que ver la superficie o la curva observada con la ecuación $f(x, y, z)=0$ ? El análisis es un instrumento ajeno a la esencia de la geometría, que por lo tanto debe ser evitado, si queremos erigir o fundar la geometría como un edificio. Mas la geometría y el análisis deben beneficiarse entre sí y servirse mutuamente como un instrumento heurístico (Cod. Ms. D. Hilbert, 60o, 1, p. 9). ${ }^{10}$

Aunque Hilbert se refiere a la cuestión de la "pureza del método" a la hora de pronunciarse a favor del método sintético o constructivo, es interesante observar que en este temprano pasaje anticipa también una suerte de principio o requisito metodológico que más tarde se volverá fundamental en su abordaje axiomático a la geometría. ${ }^{11}$ Este principio consiste en afirmar que a la hora de construir y ofrecer una fundamentación (axiomática) de la geometría, es importante que ésta sea desarrollada de un modo autónomo, esto es, con independencia de conceptos tomados de otras teorías como el álgebra, el análisis e incluso la mecánica. De este modo, uno de los objetivos fundamentales de su próximo abordaje axiomático, anticipado aquí tempranamente, será precisamente mostrar que la geometría puede ser construida desde el punto de

10 "Die Geometrie geht nicht so tief wie die Analysis. Wenn man Geometrie treibt, so muss es synthetische sein. Was hat die angeschaute Fläche oder Kurve mit eine Gleichung $f(x, y, z)=0$ zu tun? Die Analysis ist ein dem Wesen der Geometrie fremdes Hülfsmittel, welches daher vermieden werden muss, wenn man die Geometrie als Gebäude errichten oder fundieren will. Wohl dürfen sich Geometrie und Analysis gegenseitig befruchten und zu heuristischen Zwecke einander bedienen". Es difícil establecer con precisión la fecha de este pasaje. Sin embargo, corresponde a un período bien temprano. En efecto, se encuentra en las páginas iniciales del primer volumen de los "Diarios científicos" de Hilbert, que en la cubierta lleva la fecha: Leipzig, invierno de 1885 .

11 Cabe mencionar que el requerimiento de la "pureza del método" se convertirá poco después en la motivación de algunas de las investigaciones axiomáticas más fecundas de Hilbert en el campo de la geometría. Esta relación es analizada en Hallett (2008) y Arana y Mancosu (2012). 
vista de los fundamentos como una teoría autónoma o autosuficiente, que no necesita basarse en conceptos y técnicas importadas del álgebra y el análisis.

Ahora bien, detrás de estas preocupaciones por la pureza del método y el deseo de construir la geometría como una teoría autónoma es posible reconocer un problema de una vasta tradición y de enorme importancia para los fundamentos de la geometría, a saber: la determinación del papel que desempeña el número en geometría o, en otras palabras, la explicación de la relación entre la aritmética y la geometría. Aunque Hilbert no lo afirma de manera explícita en este período bien inicial, la idea de desarrollar la geometría con una teoría autónoma planteaba el problema de fondo de determinar en qué medida era posible construirla con independencia de toda consideración numérica; esta preocupación se convertirá en uno de los temas centrales de su próximo abordaje axiomático.

Cabe aclarar que este problema está inmediatamente relacionado con una serie de tesis filosóficas defendidas por Hilbert al menos en esta etapa temprana, y que resultan explícitamente señaladas en sus primeros cursos. En primer lugar, en esta etapa inicial Hilbert comparte una tesis filosófica fundamental respecto de la naturaleza de las teorías matemáticas, muy esparcida entre los matemáticos sobre todo de Alemania desde los tiempos de Gauss. Esta tesis es expresada en una célebre carta de Gauss a Bessel del 9 de abril de 1830 :

Según mi más profundo convencimiento, la teoría del espacio tiene en nuestro conocimiento a priori un lugar completamente distinto que la pura teoría de las magnitudes (reine Grössenlehre); nuestro conocimiento de la primera carece de aquel completo convencimiento de su necesidad (y también de su verdad) que es propio de la segunda. Debemos humildemente admitir que, mientras el número es sólo un producto de nuestro pensamiento, el espacio tiene además una realidad fuera de nuestro pensamiento, a la cual no podemos prescribirle a priori sus leyes (Gauss \& Bessel, 1880, p. 497). ${ }^{\mathbf{1 2}}$

De acuerdo con esta tesis, mientras que las teorías basadas en el concepto de número (aritmética, álgebra, teoría de números, análisis, etc.) debían ser consideradas teorías matemáticas puras, la geometría - y la mecánica - constituían en cambio teorías matemáticas mixtas, puesto que para su construcción no sólo basta el pensamiento puro, sino que además se requiere del material aportado por la experiencia y la intuición. Hilbert reproduce esta tesis en su curso de 1891 de la siguiente manera:

12 Para un análisis de la distinción gaussiana entre geometría y aritmética, y su influencia en la tradición matemática alemana del siglo xıx, véase Ferreirós (2006). 
La geometría es la ciencia de las propiedades del espacio, y se diferencia substancialmente de las ramas matemáticas puras, como la teoría de números, el álgebra y la teoría de funciones. Los resultados de estas disciplinas pueden ser alcanzados a través del pensamiento puro, en tanto que los hechos afirmados son reducidos por medio de claras inferencias lógicas a hechos más simples, hasta que finalmente sólo se vuelve necesario el concepto de número entero. (...) De un modo completamente distinto se comporta la geometría. No puedo nunca fundar las propiedades del espacio en la mera reflexión, tanto como no puedo reconocer de ese modo las leyes básicas de la mecánica, las leyes de la gravitación o cualquier otra ley física. El espacio no es un producto de mi pensamiento, sino que me es dado sólo a través de los sentidos [Sinne]. Para representarme sus propiedades necesito por ello de mis sentidos. Necesito de la intuición y el experimento, tanto como se los requiere para fundar las leyes físicas, donde también la materia debe sernos dada a través de los sentidos (Hilbert, 2004 [1891], p. 22-3).

Hilbert defiende la opinión, todavía compartida por muchos matemáticos hacia fines del siglo xıx, según la cual las diferentes bases epistemológicas de la aritmética y la geometría hacen que el número y los procesos recursivos resulten algo extraño o ajeno a la geometría. Desde un punto de vista epistemológico, resultaba entonces deseable lograr que el número no desempeñe un papel central en la fundamentación de la geometría. De este modo, el problema del papel del número en geometría sin dudas captó la atención de Hilbert por el problema de los fundamentos de la geometría, desde una etapa bien temprana. Este problema no sólo tenía una dimensión metodológica, asociada al requerimiento de la pureza del método, sino además una clara dimensión epistemológica, ligada al carácter peculiar de la geometría como una teoría matemática mixta. Sin embargo, la pregunta por el papel del número en la geometría se traducía en problemas matemáticos bien concretos: en primer lugar, en el estudio de cómo se realizaba la introducción del número en las distintas teorías geométricas a partir de la construcción de un sistema adecuado de coordenadas; en segundo lugar, en el análisis (axiomático) del lugar que ocupan en la estructura deductiva de estas teorías aquellos axiomas en donde los supuestos numéricos resultan más evidentes, o sea, los axiomas de continuidad. (Por ejemplo, la formulación (usual) del axioma de Arquímedes sin dudas presuponía el concepto de número entero.) Ello nos conduce a otra discusión importante de la geometría del siglo xıx, que constituyó la base de las investigaciones axiomáticas de Hilbert. 


\subsection{CoordenAdAS y GontinUIDAD}

El problema de determinar el papel que desempeñan los principios de continuidad en la estructura deductiva de la geometría euclídea fue un tema central en las investigaciones axiomáticas de Hilbert sobre los fundamentos de la geometría. Esta importancia ha sido a menudo reconocida y permite identificar una serie de problemas matemáticos concretos que en gran medida motivaron estas investigaciones. Un punto de partida de estos problemas se encuentra en la presentación de la geometría proyectiva llevada a cabo por von Staudt, cuya importante obra Geometrie der Lage (von Staudt, 1847) determinó una etapa fundamental en la historia de esta teoría geométrica. Según lo señalaba Hilbert anteriormente, uno de los méritos fundamentales de esta obra consistió en presentar a la geometría proyectiva como una teoría autónoma, que no requiere de ninguna consideración métrica para su construcción. Uno de los elementos claves del método de von Staudt consistió en renunciar al invariante proyectivo fundamental de la razón cruzada o anarmónica de cuatro puntos colineales ${ }^{\mathbf{1 3}}$ para definir la relación de proyectividad entre formas fundamentales, ${ }^{\mathbf{1 4}}$ tal como resultaba habitual en los trabajos de Möbius, Chasles y Steiner. En cambio, von Staudt proporcionó una definición puramente gráfica del conjugado armónico de un punto relativo a otros dos puntos, para lo cual utilizó una propiedad exhibida en la construcción del cuadrilátero (cuadrángulo) completo (cf. von Staudt, 1847, § 13). La unicidad de esta construcción estaba garantizada por el teorema de Desargues, que von Staudt prueba fácilmente en tanto se sitúa en el espacio. ${ }^{\mathbf{1} 5}$ Von Staudt generaliza además la noción de cuaterna armónica de modo que cubra a los elementos de un haz de rectas o un haz de planos, consiguiendo de ese modo una definición general de la correspondencia proyectiva entre dos formas fundamentales de la primera especie como una correspondencia (biunívoca) que conserva las cuaternas armónicas. ${ }^{\mathbf{1 6}}$ A esa formulación le sigue un teore-

13 La razón cruzada o anarmónica de cuatro puntos colineales $A, B, C, D$ es la cantidad (CA/CB) / (DA/DB), donde la línea en cuestión está dotada de una ordenación, de modo que esta cantidad sea positiva o negativa de acuerdo a dicha orientación. Desargues fue el primero en observar que la razón cruzada es un invariante proyectivo. Sin embargo, definido de esta manera, este concepto proyectivo básico suponía la posibilidad de medir la distancia entre dos puntos cualesquiera antes de calcular la razón cruzada, con lo cual un concepto métrico se colocaba en la base de la geometría proyectiva.

14. Von Staudt llama formas fundamentales uniformes o de la primera especie a la recta, considerada como un conjunto de puntos, al haz de rectas (en el plano) y al haz de planos.

15 En su formulación más común el teorema de Desargues afirma: sean $\triangle A B C$ y $\triangle A^{\prime} B^{\prime} C^{\prime}$ ' dos triángulos que yacen en un mismo plano; si las líneas $A A^{\prime}, B B^{\prime} y C C^{\prime}$ 'son concurrentes, los puntos de intersección de los lados correspondientes de los dos triángulos están alineados, y recíprocamente.

16 "Dos formas fundamentales uniformes se llaman proyectivas entre sí, cuando están relacionadas de tal manera que a toda forma armónica de una le corresponde una forma armónica de la otra" (von Staudt, 1847, p. 49). 
ma que, dado el papel central que cumple en el desarrollo de su teoría, fue designado poco después teorema fundamental de la geometría proyectiva: "Si dos formas fundamentales uniformes proyectivas tienen tres elementos en común, entonces todos sus elementos correspondientes son comunes" (von Staudt, 1847, p. 50).

Para demostrar el teorema fundamental von Staudt señaló que sólo se necesitaba probar el caso particular en el que las dos formas uniformes sean rectas, es decir, que dadas dos rectas cualesquiera, existe una y sólo una aplicación proyectiva que correlaciona a tres puntos cualesquiera de la primera línea con tres puntos cualesquiera de la segunda línea, en un orden dado. En tanto von Staudt caracterizó la correspondencia entre formas fundamentales sin utilizar la noción - definida apelando a consideraciones métricas - de razón anarmónica, su demostración del teorema fundamental estaba basada estrictamente en propiedades de incidencia de puntos, rectas y planos. Una consecuencia notable del método presentado por von Staudt fue que por primera vez se contó con las herramientas conceptuales necesarias como para definir coordenadas numéricas de manera puramente proyectiva (cf. Nabonnand, 2008a).

A pesar de la importancia de estos resultados, la demostración del teorema fundamental propuesta por von Staudt fue considerada posteriormente defectuosa, debido a que suponía implícitamente la propiedad de continuidad lineal. Al recurrir a la construcción de los armónicos conjugados por medio del cuadrilátero completo, la demostración original de von Staudt aseguraba la existencia de una correspondencia proyectiva, sólo en el caso de que las rectas fueran racionales. En cambio, para probar que, dados tres pares de elementos correspondientes, siempre existe una correspondencia proyectiva entre todos los elementos correspondientes, se necesitaba además que la construcción del cuarto armónico arroje como resultado una sucesión de puntos que penetre cada segmento de la recta. La demostración llevada a cabo por von Staudt suponía así un axioma que asegure la continuidad lineal, una condición que era asumida de un modo implícito (cf. von Staudt, 1847, p. 50-2; Nabonnand, 2008b, cap. 7; Voelke, 2008 , p. 251-2). Esta crítica fue formulada inicialmente por Klein (1873), aunque ciertamente de un modo confuso, en un célebre artículo que motivó un importante número de trabajos dedicados a dilucidar este problema (cf. Klein, 1873, p. 132-45). ${ }^{\mathbf{1 7}}$ El mérito de Klein fue haber notado que el problema en cuestión era de una naturaleza topológica, a la vez que puso de manifiesto la existencia de supuestos implícitos importantes no sólo en la demostración del teorema fundamental de la geometría proyectiva, sino incluso en los teoremas clásicos de Pappus (o Pascal) y Desargues.

${ }_{17}$ Poco después, Klein (1874) ensayó una solución utilizando el reciente "axioma de Cantor" que postula que a cada número real le corresponde un punto sobre la recta (cf. Cantor, 1872, p 128). En esa época, la noción de continuidad se volvió además mucho más precisa gracias a los trabajos de Weierstrass, Dedekind y Cantor sobre el conjunto de los números reales. 
La idea de que un axioma de continuidad lineal era imprescindible para demostrar el teorema fundamental de la geometría proyectiva, y por lo tanto para definir un sistema de coordenadas, fue formulada con más claridad por el matemático francés Gaston Darboux (1842-1917), en una carta dirigida a Klein y publicada luego en los Mathematische Annalen (cf. Darboux, 1880). Asimismo, a partir de las críticas iniciales de Klein al método de von Staudt, la gran mayoría de los geómetras de la época siguieron al primero en este punto, defendiendo la necesidad de postular un axioma de continuidad lineal. Sin embargo, una excepción a esta posición fue presentada por Moritz Pasch (184,3-1930) en su libro Vorlesungen über neuere Geometrie (1926 [1882]).

El trabajo de Pasch constituye la primera exposición general de la geometría proyectiva en forma axiomática, inaugurando una nueva etapa en el desarrollo de esta teoría. Por razones más bien ligadas a su concepción radicalmente empirista de la geometría que a motivos puramente matemáticos, Pasch buscó evitar que los axiomas de continuidad cumplan un papel importante en su construcción de la geometría proyectiva. ${ }^{\mathbf{8}}$ De ese modo, recién en la última sección del libro se recurre a una versión proyectiva del axioma de continuidad de Weierstrass sobre la existencia de un punto límite para mostrar cómo es posible obtener una correspondencia biunívoca entre los puntos de la línea proyectiva y los números reales (cf. Pasch, 1926, §23). ${ }^{19}$ Por el contrario, utilizando su nuevo sistema de axiomas Pasch consiguió proporcionar una prueba para el teorema fundamental en donde no se utilizaba ningún axioma de continuidad lineal, basándose en cambio en sus axiomas de congruencia, entre los cuales se encontraba una versión proyectiva del axioma de Arquímedes (cf. Pasch, 1926, p. 105).

Aunque Pasch logró mostrar que el teorema fundamental podía ser demostrado de un modo riguroso sin la necesidad de postular un axioma de continuidad lineal, su prueba resultó en cierta medida insatisfactoria, en tanto suponía que la teoría de la congruencia constituía la base en la fundamentación de la geometría proyectiva. El propio Pasch reconoció que si esta última condición quería ser evitada, entonces se debía apelar al axioma de continuidad de Weierstrass para poder probar el teorema fundamental (cf. Pasch, 1926, p. 125). ${ }^{20}$

18 "El axioma, por medio del cual Klein completó la laguna en la fundamentación de la proyectividad de Staudt, aparece en el teorema recién formulado [o sea, el axioma de Weierstrass]. Pero aceptarlo como un axioma no se corresponde sin embargo con las intuiciones a las que aquí nos sometemos. En tanto que una observación de ningún modo puede estar referida a un número infinito de cosas, la aceptación de aquel teorema no puede ser por ello mismo permitida desde nuestro punto de vista" (Pasch, 1926, p. 126.). Sobre el programa empirista de Pasch véase (Schlimm, 2010).

19 El axioma de continuidad de Weierstrass sobre la existencia de puntos límites es formulado en Pasch (1926, p. 126).

20 Sobre el procedimiento de Pasch pueden consultarse Contro (1976) y Voelke (2008). 
La cuestión central del debate era así determinar si efectivamente un axioma de continuidad lineal era imprescindible para probar el teorema fundamental de la geometría proyectiva, y del mismo modo, para la introducción de un sistema de coordenadas adecuado. Entre los geómetras que siguieron la propuesta de Pasch e intentaron desarrollar la geometría proyectiva reduciendo a un mínimo la utilización de principios de continuidad, se destacan las contribuciones de dos autores particularmente importantes para Hilbert: Hermann Wiener (1857-1939) y Friedrich Schur (18561932). En una conferencia impartida ante laAsociación de Matemáticos Alemanes (DMV) en septiembre de 1891, Wiener sostuvo que sería posible utilizar los axiomas de Desargues y Pascal - a los que bautizó “teoremas de incidencia” [Schliessungssätze] para probar el teorema fundamental de la geometría proyectiva (cf. Wiener, 1891, p. 47 ). Dado que estos teoremas de incidencia no requerían en principio de la aceptación de ningún axioma de continuidad, Wiener propuso que toda la geometría proyectiva podía ser construida sin apelar a este tipo de condiciones. En la conferencia de 1891 no encontramos demostración alguna para estas dos afirmaciones, sin embargo dos años después, en una segunda conferencia en donde se analizan las implicaciones de estas ideas para la geometría afín y euclídea, Wiener probó que el teorema de Pascal podía ser demostrado utilizando sólo los axiomas de congruencia de Pasch y el axioma de las paralelas, y sin apelar por lo tanto a ningún axioma de continuidad, en particular al axioma de Arquímedes (cf. Wiener, 1893). En una nota sugirió además que de esta misma manera podía ser demostrado el teorema fundamental de la geometría proyectiva (cf. Wiener, 1893, p. 72).

Esta última sugerencia fue investigada por Schur, quien en 1898 logró demostrar el teorema de Pascal en el plano exclusivamente sobre la base de los teoremas de congruencia en el espacio, y utilizando luego este teorema, proporcionó una nueva demostración del teorema fundamental (cf. Schur, 1898). Los resultados alcanzados por Schur impresionaron notablemente a Hilbert, quien inmediatamente hizo de la tarea de determinar el papel de los principios de continuidad - particularmente el de Arquímedes - uno de los temas centrales de su nuevo abordaje axiomático formal a la geometría. Más precisamente, el hecho de que los teoremas de Desargues y Pascal cumplían un papel central en el procedimiento de von Staudt para introducir coordenadas en la geometría proyectiva era como hemos visto una cuestión bien conocida en la última década del siglo xıx. Sin embargo, lo que nadie antes de Hilbert fue capaz de percibir fue la posibilidad de utilizar estos teoremas para introducir coordenadas en la geometría euclídea desde dentro. Ello significaba construir nuevos puentes, por medio del método axiomático, entre las geometrías sintéticas y las geometrías analíticas construidas sobre diversos cuerpos de números. 


\section{Geometría y número: el PRograma de Hilbert}

En la sección anterior hemos señalado que las discusiones metodológicas entre los geómetras sintéticos puros y los geómetras analíticos constituyeron un trasfondo importante en el abordaje axiomático a la geometría llevado a cabo por Hilbert. Especialmente, nuestro matemático entendía que este debate, intensamente mantenido en Alemania en la primera mitad del siglo xix, planteaba el problema de fondo de proporcionar una explicación adecuada respecto del papel del número en geometría. Dicho de otro modo, esta controversia planteaba en definitiva el problema de determinar en qué medida era necesaria, y cómo se justificaba desde el punto de vista de los fundamentos, la introducción del número en geometría. La preocupación por este problema se tradujo en el hecho de que, en Fundamentos de la geometría, Hilbert impone ciertas condiciones o cuidados especiales en la introducción de un sistema de coordenadas numéricas para su teoría geométrica. Más precisamente, el procedimiento propuesto allí para la construcción de un sistema de coordenadas denota una preocupación muy especial respecto de la relación entre la geometría euclídea elemental y la estructura (algebraica) de la geometría analítica. Sobre esta cuestión en particular, sus cursos resultan sumamente esclarecedores, en tanto presentan numerosas reflexiones respecto de la importancia metodológica y epistemológica de este problema, y en particular, sobre cómo el método axiomático formal podía contribuir enormemente a su elucidación.

\subsection{LA INTRODUCGIÓN DEL NÚMERO EN 1893/1984}

El primer abordaje axiomático a la geometría llevado a cabo por Hilbert lo constituyen las notas de clases para el curso "Los axiomas de la geometría" (Hilbert, 2004, [1893/ 1894]), dictado en Königsberg en el semestre de invierno de 1893/1894. ${ }^{\mathbf{2 1}}$ El método axiomático formal no está allí completamente desarrollado como en sus cursos posteriores en 1898/9, o como en la primera edición de Fundamentos de la geometría (Hilbert, 1899). ${ }^{22}$ Por un lado, Hilbert no lleva adelante en este curso una investigación axiomática tal como la define previamente en su anterior curso de 1891, i.e., "una investigación sistemática de aquellas geometrías que surgen cuando uno o más axiomas son dejados de lado o reemplazados por su negación" (Hilbert, 2004 [1891], p. 22). Por otro lado, tampoco se llevan a cabo aquí investigaciones "metageométricas", o sea, un

21 El curso fue presentado inicialmente para el semestre de verano de 1893, pero el escaso número de alumnos interesados hizo que se postergara para el semestre siguiente. Hilbert aprovechó el aplazamiento para completar sus notas con nuevas lecturas, especialmente Pasch (1926). Sobre el origen y características de estas notas, véase Majer y Hallett (2004, cap. 2) y Toepell (1986).

22 En adelante llamaremos Festschrift a la primera edición de Fundamentos de la geometría (Hilbert, 1899). 
estudio de la independencia de los axiomas entre sí y de la consistencia del sistema axiomático, utilizando el método de proporcionar distintas interpretaciones o "modelos" de los axiomas. ${ }^{23}$ Sin embargo, estas notas de clases constituyen el inicio de un análisis axiomático, de donde se sigue que preparan el camino para los posteriores tratamientos metageométricos.

En lo que se refiere al problema de la introducción del número es posible realizar dos observaciones. En primer lugar, en estas notas Hilbert reconoce por primera vez de un modo explícito la importancia metodológica y epistemológica que reviste este problema para la construcción sistemática de la geometría y para un examen de sus fundamentos. En segundo lugar, la estructura y organización de este curso revela que, en este primer ensayo axiomático, Hilbert adopta la estrategia - posteriormente por él criticada de introducir el número en la geometría lo más rápido posible. Su objetivo parece ser aquí mostrar cómo es posible introducir coordenadas en la geometría sin apelar a consideraciones de congruencia, para después exhibir cómo la geometría hiperbólica y elíptica pueden ser desarrolladas sobre estos fundamentos mínimos; por esta razón el axioma euclídeo de las paralelas es el último en ser introducido (cf. Hilbert, 2004 [1893/1894]).

Hilbert organiza su exposición de la siguiente manera: en primer lugar presenta el grupo de axiomas de incidencia o "existencia”, como se los designa allí, que establecen las relaciones de incidencia entre puntos, líneas y planos. En segundo lugar, formula el grupo de axiomas de "posición", que resultan adecuados para describir las relaciones de ordenación en la geometría euclídea. En este grupo de axiomas se nota claramente la influencia del libro Lecciones de geometría moderna (1882) de Moritz Pasch, en tanto cinco de los seis axiomas allí formulados son tomados de aquel libro. Hilbert hace entonces un breve paréntesis en su exposición para introducir una serie de conceptos proyectivos básicos, entre ellos el concepto de “separación” de cuatro puntos colineales. Por otra parte, otro concepto fundamental allí introducido es la noción de "cuaterna armónica", utilizado como dijimos por von Staudt para definir la noción misma de proyectividad. Hilbert analiza la construcción clásica del cuarto elemento armónico, siguiendo el procedimiento basado en las técnicas desarrolladas por von Staudt, i.e., la construcción del cuadrilátero completo (cf. Hilbert, 2004 [1893/1894], p. 81-2). Ahora bien, esta construcción armónica no sólo permite definir varios conceptos centrales de la geometría proyectiva, sino que además hace posible la correlación entre los

23 Quizás ésta haya sido una razón por la cual en un primer momento Hilbert consideró sus investigaciones axiomáticas como "poco interesantes" y "estériles”, desde un punto de vista matemático. Esta opinón se encuentra en una carta a su colega y amigo, Adolf Hurwitz (1859-1919): "por cierto mi curso sobre los axiomas de la geometría no me ha resultado, por lo menos por ahora, para nada edificante. Siempre lo mismo: si se debe tomar esto o aquello como axioma; siempre el mismo tono insípido, sin la vívida frescura de los nuevos resultados" (Hilbert a Hurwitz, 13 de junio de 1894, apud Toepell, 1986, p. 100). 
puntos de una línea y los números reales. Hilbert emprende inmediatamente esta tarea con el objetivo de exhibir cómo se pueden introducir coordenadas sobre esta base mínima de axiomas de incidencia y orden, por lo tanto, antes de establecer los axiomas de congruencia. Este procedimiento es estudiado en una sección titulada "La introducción del número" (cf. Hilbert, 2004, [1893/1894], p. 85-93).

En el comienzo de esa sección, Hilbert destaca la importancia epistemológica que recae sobre la introducción del número en geometría:

En todas las ciencias exactas recién se alcanzan resultados precisos cuando el número es introducido. Observar cómo ello ocurre tiene un gran significado epistemológico (erkenntnisstheoretisch) (Hilbert, 2004, [1893/1894], p. 85).

A continuación utiliza las técnicas desarrolladas por von Staudt para mostrar que esta construcción armónica de cuatro puntos colineales permite encontrar un único punto sobre la recta para cada número racional (positivo) (cf. Hilbert, 2004, [1893/ 1894], p. 85-8). Más aún, utilizando esta misma construcción, Hilbert muestra cómo es posible asignarle a cada punto sobre la recta un (único) número real (positivo). ( $\mathrm{Si}$ además se define un sentido sobre la línea, entonces también se pueden cubrir los números negativos.) Sin embargo, reconoce inmediatamente que para que la afirmación recíproca se cumpla, es decir, para que a cada número real (positivo) le corresponda un punto sobre la línea, es necesario agregar un nuevo axioma que garantice la continuidad lineal. Hilbert formula entonces un axioma de continuidad que establece la existencia de un punto límite para una sucesión monótona creciente y acotada superiormente de puntos sobre la línea, lo cual garantiza la correspondencia uno-a-uno entre los puntos de una línea y los números reales. ${ }^{24}$

Ahora bien, la introducción de este axioma de continuidad es realizada muy rápidamente y de ningún modo es analizada en detalle. Por ejemplo, Hilbert limita su análisis de la introducción del número a establecer esta correspondencia uno-a-uno con los números reales, mientras que en cambio no se preocupa por investigar las propiedades algebraicas de los "análogos geométricos" a los números introducidos, es decir, las propiedades de un cuerpo. Estas propiedades son las que permiten aplicar

24 “Axioma de continuidad. Sea $P_{1}, P_{2}, P_{3}, \ldots$ una sucesión infinita ordenada de puntos sobre una recta. Si todos los puntos se encuentran de un mismo lado respecto de un punto $A$, entonces siempre existe un y sólo un punto $P$ tal que todos los puntos de la sucesión se encuentran de un mismo lado respecto de $P$, y al mismo tiempo no existe ningún punto entre $P$ y todos los puntos de la sucesión. $P$ se llama el punto límite" (Hilbert, 2004, [1893/1894], p. 92). Hilbert reproduce nuevamente este axioma en una carta a Klein, fechada del 14, de agosto de 1894, y publicada más tarde en los Mathematische Annalen (Hilbert, 1999 [1895]). Una versión similar de este axioma - conocido también como principio de Bolzano-Weierstrass sobre la existencia de puntos límites - se encuentra previamente en Pasch (1926, p. 125-6), de quien Hilbert probablemente tomó el axioma. 
los números para medir y describir las propiedades de los objetos geométricos (la línea, el rectángulo, el círculo, etc.).

Luego, en este primer estudio axiomático, Hilbert no se interesa en ningún momento por la cuestión de hasta dónde puede ser desarrollada la geometría (euclídea) elemental, antes de utilizar algún postulado de continuidad, un tema que posteriormente se volverá uno de los elementos claves en Fundamentos de la geometría (Hilbert, 1899). Estos dos puntos revelan un importante cambio de actitud, en su siguiente curso de 1898/1899, respecto de cómo podía ser desarrollada la introducción del número en geometría. El cambio de actitud de Hilbert quizás se explica en virtud de que, en este primer momento, no estaba completamente convencido de que fuera posible realizar enteramente la introducción de un sistema de coordenadas sin apelar a ningún axioma de continuidad. Una alteración significativa hallada por M. Toepell en este mismo manuscrito de 1893/1894 sugiere esta hipótesis; a saber, en un pasaje tachado Hilbert aclara: "probar los resultados de Wiener si son correctos, lo cual me parece dudoso" (Toepell, 1986, p. 78; Wiener, 1891).

\subsection{Puentes axiomáticos: LA Introdugaión del número en 1898/1899}

El próximo curso que Hilbert dedica a los fundamentos de la geometría corresponde al semestre de invierno de 1898/1899. Uno de los aspectos más importantes de este curso es que constituye el antecedente inmediato del Festschrift (Hilbert, 1899; cf. Majer \& Hallett, 2004, cap. 4). Como un objetivo central de este nuevo análisis axiomático, Hilbert se propone investigar cómo pueden y deben ser introducidos los números en la geometría; más aún, destaca ahora que el método axiomático puede ser de gran ayuda en este respecto, en tanto puede contribuir a profundizar nuestra comprensión de las conexiones conceptuales entre la geometría sintética y la geometría analítica. Hilbert resalta además que en la resolución de este problema se puede apreciar con claridad la fecundidad matemática del método axiomático formal. Esta cuestión aparece sugerentemente indicada en una versión de este curso elaborada por el propio Hilbert (2004 [1898a]), en donde en cierta medida critica el modo en que en su curso anterior (Hilbert, 2004 [1893/1894]) había sido tratada la introducción del número:

Con estas premisas la geometría se ha vuelto inmediatamente un cálculo (Rechenkunst). Es claro que utilizando ángulos rectos, paralelas, longitudes y distancias estamos suponiendo todo lo que es fundamental en la geometría elemental. Así, hemos tomado la vía en la que la introducción del número en la geometría es alcanzada tan rápido como sea posible y a cualquier precio. Ahora, en todas las ciencias la introducción del número es de hecho el objetivo más noble. 
Es posible medir el progreso de las ciencias naturales, o de una rama de la ciencia natural, en función del grado en el que el número ha sido introducido. Sin embargo, si la ciencia no quiere caer presa de un formalismo estéril (unfruchtbarer Formalismus), entonces deberá reflexionar sobre sí misma en una fase posterior de su desarrollo y, por lo menos, examinar cómo se ha logrado la introducción del número (Hilbert, 2004 [1898a], p. 223; énfasis mío).

Hilbert reconoce de esta manera la importancia, no sólo para la matemática sino también para todas las ciencias en general, de investigar cómo es llevada a cabo la introducción del número. En el caso particular de la geometría euclídea, la vía que se propone desarrollar es la siguiente:

Por lo tanto, en nuestro curso la introducción del número en la geometría aparecerá directamente en la última etapa como un objetivo final, que viene a coronar el edificio de la geometría hasta allí construido (Hilbert, 2004 [1898a], p. 223).

Al afirmar que la introducción del número será realizada en una última etapa como un "objetivo final", Hilbert expresa su interés en que esta introducción no sea realizada como una imposición desde fuera, como ocurre en la geometría analítica, sino desarrollando axiomáticamente una estructura equivalente a la de los números reales desde dentro, o sea, de manera puramente geométrica. Asimismo, esta dilación en la introducción del número le permitirá investigar cuáles son los recursos algebraicos disponibles dentro de la estructura de la geometría sintética, independientes de la introducción de presupuestos específicamente numéricos o de continuidad. Por ejemplo, una tarea emprendida en este curso, y luego en el Festschrift, consistió en analizar qué axiomas son responsables de la presencia de la estructura de un cuerpo ordenado sobre la línea. Al mismo tiempo, Hilbert reconoció que un importante beneficio que conlleva este tipo de abordaje es que permite descubrir nuevas e importantes conexiones entre la geometría y la aritmética:

Pero investigar nuevamente los elementos de la geometría euclídea no es sólo de una necesidad práctica y epistemológica, sino que espero también que los resultados que obtendremos valdrán el considerable esfuerzo. Seremos conducidos a una serie de problemas en apariencia simples, pero en verdad bien profundos y difíciles. Llegaremos a reconocer preguntas completamente nuevas y, en mi opinión muy fructíferas, acerca de los elementos de la aritmética y los elementos de la geometría, y de esa manera llegaremos a proporcionar nuevamente un fundamento para la unidad de la matemática (Hilbert, 2004, [1898a], p. 223; énfasis mío). 
Vemos aquí que una parte esencial de la empresa hilbertiana de construir axiomáticamente la geometría consistía en mostrar que esta disciplina podía ser desarrollada de manera independiente a la aritmética y el análisis. Esa tarea procedía en dos direcciones, ambas conectadas con su "aritmética de segmentos" (Streckenrechnung). En primer lugar, Hilbert demuestra que muchos resultados importantes de la geometría elemental pueden ser alcanzados sin apelar a postulados de continuidad y, además, que estos principios de continuidad pueden ser formulados de un modo puramente geométrico. En parte, Hilbert desarrolla por esta razón su aritmética de segmentos, que imita el comportamiento de los números racionales de un modo puramente geométrico. Este cálculo podía ser entonces utilizado para elaborar una nueva teoría de las proporciones, a la cual se podía acudir para formular el axioma de Arquímedes, el único axioma de continuidad utilizado en el Festschrift.

En segundo lugar, con su aritmética de segmentos Hilbert revela cómo es posible construir, de manera puramente geométrica, una estructura algebraica equivalente a un cuerpo ordenado, y a partir de allí cómo introducir coordenadas en la geometría "desde dentro". Es decir, Hilbert consigue mostrar que los segmentos lineales, junto con las operaciones definidas para ellos, pueden ser utilizados como la base de cuerpos adecuados para llevar a cabo una coordenatización interna de la geometría, y de ese manera, exhibir que, en cierto modo, la geometría analítica es posible sin tener que recurrir a la imposición de cuerpos numéricos "desde fuera”. Estas innovaciones técnicas le permitieron mostrar que en ningún momento, en la construcción de la geometría, estamos forzados a suponer que la geometría debe ser construida sobre una variedad de números, una suposición muy común en el siglo xIX.

De este modo, por medio de su análisis axiomático Hilbert logró mostrar cómo la geometría podía ser construida como una teoría matemática pura, pero que no dependía esencialmente de ningún tipo de número. Este resultado constituía un claro contraejemplo para la tesis clásica de Kronecker, rechazada siempre por Hilbert, según la cual sólo podía considerarse como teorías matemáticas puras a aquellas teorías que en última instancia podían ser inmediatamente reducidas a la teoría de los números naturales (cf. Blumenthal, 1922, p. 68). Sin embargo, es importante señalar que Hilbert no consideraba su reconstrucción axiomática de la geometría sintética como una manera de probar la pretendida superioridad de la geometría pura, sino más bien como un modo de unir o trazar un puente entre las geometrías sintéticas axiomatizadas y la geometría analítica. Hilbert sugiere precisamente este rasgo de su empresa axiomática de la siguiente manera:

A partir de lo dicho se esclarece la relación de este curso con aquellos sobre geometría analítica y geometría proyectiva (sintética). En ambas disciplinas las 
preguntas fundamentales no son tratadas. En la geometría analítica se comienza con la introducción del número; por el contrario nosotros habremos de investigar con precisión la justificación para ello, de modo que en nuestro caso la introducción del número se producirá al final. En la geometría proyectiva se apela desde el principio a la intuición, mientras que nosotros queremos analizar la intuición, para reconstruirla, por decirlo de algún modo, en sus componentes particulares (einzelne Bestandteile) (Hilbert, 2004 [1898b], p. 3o3; énfasis mío).

Empero cabe aclarar que el requerimiento de Hilbert según el cual la geometría debe ser construida independientemente del análisis y la aritmética, convive con la utilización de interpretaciones aritméticas y analíticas para mostrar que los diversos axiomas empleados son independientes entre sí. La utilización de conceptos y técnicas analíticas y algebraicas no es rechazada en absoluto por Hilbert, sino que más bien está reservada para el nivel metageométrico, en donde constituye una herramienta imprescindible (cf. Hilbert, 2004 [1898a], p. 222).

\section{UNA ARITMETIZAGIÓN INTERNA DE LA GEOMETRÍA}

Hilbert pretende lograr una presentación axiomática de la geometría en la que los números no son introducidos "desde fuera", como elementos externos o exógenos, sino que en cambio son introducidos "desde dentro", es decir, de un modo puramente geométrico. Para alcanzar este objetivo, elabora de manera puramente geométrica una aritmética de segmentos lineales, cuyas operaciones coinciden con las reglas usuales de los números racionales. Exclusivamente por medio de construcciones geométricas, Hilbert define las operaciones de suma y multiplicación de segmentos y muestra cómo se puede construir de ese modo un conjunto con la estructura de un cuerpo ordenado, cuando se toman como los elementos positivos de este conjunto a las clases de equivalencia de segmentos lineales (módulo congruencia). La novedad de este procedimiento consiste en que, en lugar de utilizar una noción "preexistente" de número, como los números racionales o los números reales, Hilbert genera de manera puramente geométrica un conjunto cuya estructura se corresponde a la de un cuerpo numérico (abstracto), al cual se podía acudir luego para definir un sistema de coordenadas. En otras palabras, Hilbert logra mostrar cómo es posible llevar a cabo una aritmetización interna de la geometría.

Para poder apreciar el alcance del proyecto de Hilbert, resultará útil comparar rápidamente su presentación axiomática de la geometría en Fundamentos de la geometría (1899), con la estructura de los Elementos de Euclides. Me ocuparé de ello a continuación. 


\subsection{Los Grundlagen de Hilbert y los Elementos de Euclides}

Un aspecto relevante a la hora de analizar Fundamentos de la geometría descansa en el hecho de que, como lo ha observado David Rowe (2000), desde el punto de vista de la estructura la geometría euclídea era en 1898 más parecida a los Elementos de Euclides que a los Grundlagen de Hilbert (cf. Rowe, 2000, p, 68). Estas diferencias estructurales están íntimamente ligadas a la aritmética de segmentos elaborada por éste último. ${ }^{25}$

Como es bien sabido, en los primeros cuatro libros de los Elementos, Euclides desarrolla una teoría geométrica pura sin números. No encontramos en estos libros una noción de longitud de un segmento lineal, ni de amplitud de un ángulo, ni números asignados a las figuras planas en el estudio de las áreas, sino que todas las figuras geométricas son estudiadas apelando a la noción no definida de congruencia, que intenta expresar que dos figuras (segmentos, ángulos, áreas) tienen el mismo "tamaño". La estrategia de Euclides en los libros I-IV consiste en probar la mayor cantidad de teoremas posibles apelando a los teoremas de congruencia. El libro I trata de las figuras rectilíneas congruentes y culmina con el teorema de Pitágoras. El libro II introduce una suerte de álgebra geométrica de segmentos y rectángulos, cuyas propiedades están basadas en los teoremas de congruencia; y en los libros III-IV se aplican los resultados de los libros previos a la teoría de los círculos y los polígonos regulares. ${ }^{26}$ Sin embargo, esta estrategia enfrenta una dificultad cuando Euclides debe ocuparse de la teoría de los triángulos semejantes, o sea, triángulos cuyos lados correspondientes no son iguales, pero tienen una razón común entre sí. La teoría de la congruencia de triángulos puede ser utilizada sin problemas para estudiar la semejanza de triángulos, en el caso de que las razones de los lados correspondientes sean números enteros, o incluso racionales. En cambio, si las razones entre los lados correspondientes de dos triángulos son números irracionales, resulta claramente problemático expresar que la razón entre la longitud de los lados correspondientes de los triángulos es la misma, si dichas longitudes no pueden ser expresadas numéricamente. Para superar esta dificultad, Euclides interrumpe su exposición "puramente geométrica" y presenta en el libro V de los Elementos la célebre "teoría de las proporciones", atribuida por Proclo a Eudoxio de Cnidos. ${ }^{27}$

Un rasgo central de la teoría de las proporciones de Eudoxio es que allí no se define qué es una razón o proporción entre dos magnitudes, sino en cambio cuando dos razones son iguales entre sí, o cuando una es mayor o menor que la otra. En efecto,

25 Para una comparación de la estructura de los Elementos de Euclides y los Grundlagen de Hilbert, pueden verse Hartshorne (2000, cap. 1-4) y Greenberg (2000, cap. 1-4).

26 Sobre la estructura de los Elementos, puede consultarse el clásico trabajo de Mueller (1981).

27 Sobre el origen de la teoría de las proporciones del libro V, véase Heath (1956). 
esta noción es formulada en la definición $\mathrm{V}$, considerada generalmente como la definición más importante del libro V:

Dícese que la razón de una primera magnitud a una segunda es igual a la de una tercera a una cuarta, cuando las primeras y las terceras igualmente multiplicadas o al mismo tiempo superan, o al mismo tiempo son iguales o al mismo tiempo son inferiores que las segundas y cuartas igualmente multiplicadas (Elementos, V, def. 5).

Es usual explicar el contenido de esta definición, utilizando una notación algebraica moderna, de la siguiente manera: dos magnitudes (segmentos lineales, áreas, volúmenes, etc.) tienen la misma razón respecto de otras dos (en símbolos $a: b=c: d$ ) si tomando $m$ múltiplos (enteros positivos) de $a$ y c y $n$ múltiplos (enteros positivos) de by $d$, se tiene que:

$$
m a \lesseqgtr n b \text { síy sólo si } m c \lesseqgtr n d \text {. }
$$

De la definición anterior se sigue también que si $a: b=c: d$, entonces existen dos múltiplos (enteros positivos) $m, n$ tal que $m a>n b$, pero $m c \leq n d$. Sin embargo, estas últimas desigualdades plantean una dificultad. Si se quiere probar para $a<b$ se cumple $a: a>a: b$, entonces es necesario buscar dos $m, n$ (enteros positivos) tales que $m a>n a$, pero $m a \leq n b$. Luego, si $m=n+1$, entonces se tiene que

$$
(n+1) a \leq n d
$$

o bien,

$$
a \leq(b-a) n
$$

Ello significa que para las magnitudes $a$ y $d=(b-a)$, se debe encontrar un $n$ (entero positivo) tal que

$$
n d \geq a .
$$

y éste es precisamente el axioma de Arquímedes, que en el quinto libro de los Elementos aparece sugerido en la definición $4 \cdot{ }^{28}$

28 "Se dice que guardan razón entre sí las magnitudes que, al multiplicarse, pueden exceder una a otra" (Elementos, V, def. 4). 
Tras desarrollar íntegramente la teoría de las proporciones de las magnitudes generales de un modo "abstracto" en el libro V de los Elementos, en el libro siguiente Euclides la aplica a la geometría plana, desarrollando la teoría de los triángulos semejantes. Hilbert advierte en sus notas de clases que el resultado más importante de esta teoría, utilizado prácticamente en todas las demostraciones subsiguientes, es presentado en la proposición VI.2, a veces también referida como el teorema de Tales (cf. Hilbert, 2004 [1898a], p. 274--5; Hilbert, 2004 [1898b], p. 363).

Si se traza una recta paralela a uno de los lados de un triángulo, cortará proporcionalmente los lados del triángulo. Y si se cortan proporcionalmente los lados de un triángulo, la recta que une los puntos de sección será paralela al lado restante del triángulo (ver Fig. 1).

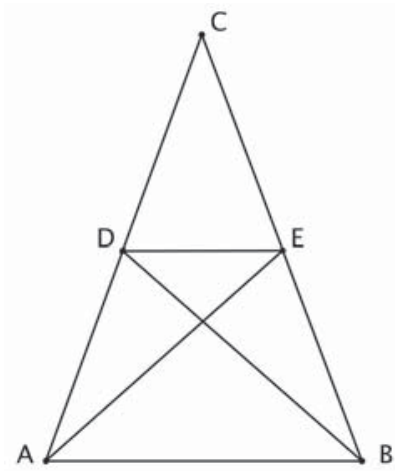

Figura 1. Elementos, Proposición VI. 2.

La demostración que propone Euclides es una de las más ingeniosas de todo los Elementos y utiliza la teoría del área, desarrollada de un modo rudimentario en el libro I. Luego, Hilbert identifica al menos dos problemas con la teoría de las proporciones y de los triángulos semejantes que desarrolla Euclides en los Elementos (cf. Hilbert, 2004 [1898a], p. 275; 2004 [1898b], p. 363). En primer lugar, la teoría de las proporciones del libro $\mathrm{V}$ no posee el mismo carácter puramente geométrico que los cuatros libros anteriores; se trata de una teoría de una naturaleza aritmética, más que geométrica. Euclides no explica en ningún momento qué es una razón o una proporción geométricamente, sino que define aritméticamente la identidad entre razones, o sea, como una "ecuación numérica". ${ }^{29}$ En consecuencia, la teoría de los triángulos semejantes del libro VI se basa en dos teorías con fundamentos bien distintos: uno geométrico y el

29 Existe una vastísima literatura en torno a cómo debe ser interpretada la relación entre números, magnitudes, razones y proporciones en los Elementos de Euclides. Puede consultarse, por ejemplo, Mueller (1981) y GrattanGuinness (1996). 
otro aritmético; desde el punto un punto de vista metodológico, ésta era una consecuencia que Hilbert prefería evitar.

En segundo lugar, la teoría de las proporciones de Eudoxio supone la validez del axioma de Arquímedes. Dado que un objetivo central de Hilbert era prescindir lo más posible de los axiomas de continuidad, la teoría de las proporciones del libro V resultaba por ello inadecuada. ${ }^{3 \circ}$ Remediar estas dificultades fue una contribución muy importante de Hilbert a los fundamentos de la geometría euclídea. Por un lado, el matemático alemán elaboró una nueva teoría de las proporciones exclusivamente sobre la base de su aritmética de segmentos, construida de manera puramente geométrica e independientemente del axioma de Arquímedes. Por otro lado, aplicó esta nueva teoría de las proporciones para desarrollar la teoría de los triángulos semejantes y del área. Hilbert llevó a cabo una unificación de dos teorías que, anteriormente, estaban basadas en fundamentos distintos, dando al mismo tiempo una respuesta al problema de la introducción del número en geometría.

\subsection{El GÁlgulo de segmentos [Streckenrechnungen] de Hilbert}

Los resultados geométricos mencionados en las secciones anteriores se encuentran en los capítulos 3-5 de Fundamentos de la geometría (1899). En el capítulo 3, Hilbert construye una aritmética de segmentos basada en el teorema de Pascal, presenta su nueva teoría de las proporciones y de los triángulos semejantes, e indica cómo es posible definir un sistema de coordenadas (cartesianas) utilizando esta aritmética de segmentos. El capítulo 4, está dedicado a la teoría euclídea del área, que Hilbert reconstruye utilizando su teoría de las proporciones y la aritmética de segmentos desarrollada en el capítulo anterior, y por lo tanto, sin utilizar ningún axioma de continuidad. Finalmente, el capítulo 5 se ocupa del teorema del Desargues y de la aritmética de segmentos que se puede construir basándose en este teorema. En dicho capítulo se demuestra que, mientras que la aritmética de segmentos asociada al teorema de Pascal satisface todas las propiedades de un cuerpo ordenado, la aritmética de segmentos asociada al teorema de Desargues carece de la propiedad conmutativa bajo la multiplicación. En lo que sigue me concentraré en la aritmética de segmentos construida en el capítulo 3, que contiene los resultados más interesantes para el problema que venimos analizando.

Dado que Hilbert se plantea explícitamente el objetivo de mostrar que las operaciones definidas para los segmentos lineales cumplen con todas las propiedades usualmente asociadas con la aritmética de los reales, es claro que para ello era necesario contar una axiomatización precisa de la estructura de un cuerpo ordenado, que permi-

3o Otra dificultad en la teoría de los triángulos semejantes de Euclides es que presupone algunos resultados de la teoría del área, la cual no fue sin embargo satisfactoriamente tratada en el libro I (cf. Hartshorne, 2000). 
ta distinguir qué propiedades comparten y qué propiedades no comparten ambos cuerpos. Éste es el origen de su conjunto de axiomas para un "conjunto de números complejos", que constituye de hecho el primer sistema axiomático para un cuerpo ordenado arquimediano. ${ }^{3 \mathbf{1}}$ Hilbert presenta una primera versión de este sistema de axiomas en su curso de 1898/1899, el cual es reproducido en la sección §13 del capítulo III del Festschrift. (cf. Hilbert, 1899, §13). Poco después, en su conferencia de Munich "Sobre el concepto de número" (Hilbert, 1900a), el sistema de axiomas original es complementado con su famoso "axioma de completitud" (Vollständigkeitsaxiom), con lo cual se obtiene la primera caracterización axiomática de un cuerpo ordenado completo. ${ }^{\mathbf{3 2}}$

Tras presentar esta caracterización axiomática de la estructura de un cuerpo ordenado (completo), el próximo paso consiste en proporcionar una demostración de un caso especial del teorema de Pascal (más conocido como teorema de Pappus) para las secciones cónicas, de notable importancia en la geometría proyectiva. Se trata de una versión afín del teorema, que Hilbert enuncia de la siguiente manera:

Teorema de Pascal. Sean dados dos conjuntos de puntos $A, B, C$ y $A^{\prime}, B^{\prime}, C^{\prime}$ situados respectivamente sobre dos rectas que se intersecan, de tal manera que ninguno de ellos se encuentra en la intersección de estas líneas. Si $C B^{\prime}$ es paralelo a $B C^{\prime}$ y $C A$ ' es también paralelo a $A C^{\prime}$, entonces $B A^{\prime}$ 'es paralelo a $A B^{\prime}$ (Hilbert, 1899, p. 28). (ver Fig. 2)

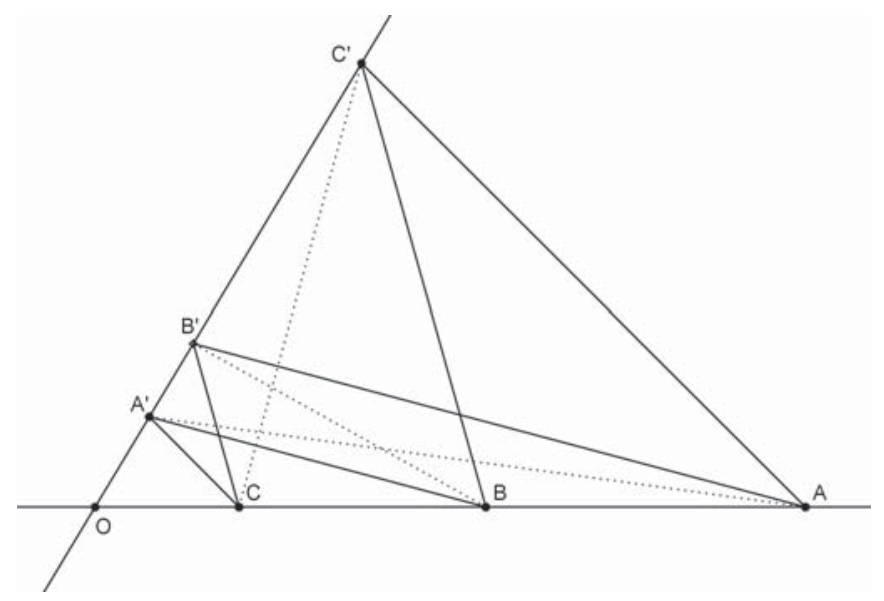

Figura 2. Versión afín del teorema de Pascal para las secciones cónicas.

31 En sus notas de clases, Hilbert aclara que por un sistema de números complejos entiende a todo sistema de números que, al igual que los números complejos, no satisface todos los axiomas para los números reales. De acuerdo con esta definición, el cuerpo de números algebraicos son así ejemplos de sistemas de números complejos (cf. Hilbert, 2004 [1902], p. 564).

32 Sobre la inclusión del axioma de completitud en ediciones posteriores de Fundamentos de la geometría (1899) puede verse Giovannini (2013). 
La importancia de la demostración del teorema de Pascal proporcionada por Hilbert consistía en que apelaba a los axiomas de congruencia (IV) y los axiomas de orden (II) e incidencia en el plano (I, 1-2), lo cual era un argumento técnicamente difícil de llevar a cabo. En consecuencia, dicha demostración no hace uso de ningún postulado de continuidad, en particular, del axioma de Arquímedes. ${ }^{33}$ El teorema de Pascal proporciona asimismo lo necesario para construir una aritmética de segmentos lineales, en donde son válidas todas las operaciones "de los números reales" (cf. Hilbert, 1899, p. 32). En primer lugar, Hilbert aclara que, en el cálculo de segmentos que se presentará a continuación, la palabra "igual” y el signo "=" serán utilizados en lugar de la palabra "congruentes" y el signo "三” (cf. Hilbert, 1899, p. 33). Ello significa que las operaciones de suma y multiplicación serán definidas para clases de equivalencia de segmentos lineales (módulo congruencia). La primera operación en ser definida es la suma o adición de segmentos lineales. Hilbert define esta operación de una manera muy simple, de acuerdo a como era habitual caracterizar esta operación de un modo puramente geométrico:

Definición. Si $A, B, C$ son tres puntos sobre una línea y $B$ se encuentra entre $A$ y $C$, entonces decimos que $c=A C$ es la suma de los segmentos $a=A B$ y $b=B C$, y establecemos que

$$
c=a+b
$$

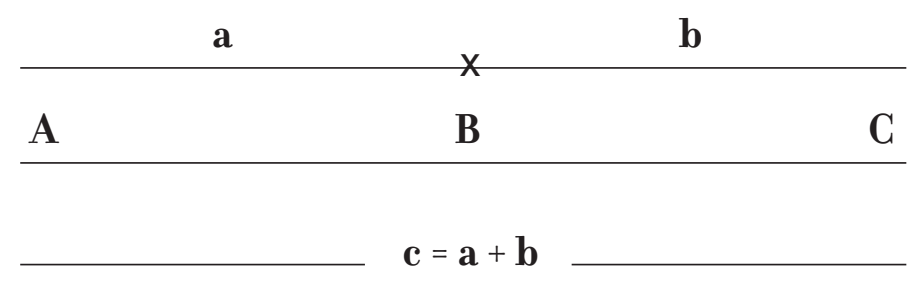

Figura 3. Suma de segmentos lineales. Adaptado de Hilbert (1899, p. 33).

Dada esta definición de la suma de segmentos lineales, los axiomas de congruencia para segmentos (III, 1-3) permiten probar muy fácilmente las leyes asociativa y conmutativa para la adición. Dada su simplicidad, Hilbert no proporciona una demostración de estas propiedades. Estas pruebas pueden encontrarse, por ejemplo, en Hartshorne (2000, p. 168-9). En cuanto a la definición del producto de segmentos

33 Muy poco tiempo antes, Schur (1898) había proporcionado una prueba del axioma de Pascal sin utilizar el axioma de Arquímedes, basándose sin embargo en todos los axiomas de incidencia, orden y congruencia (I-II, IV). Sobre la influencia de este resultado de Schur en Hilbert, véase Toepell (1985). 
lineales, Hilbert recurre a una construcción geométrica muy simple, similar a la utilizada por primera vez por Descartes para definir la multiplicación de segmentos. La construcción procede de la siguiente manera: Sean $a$ y $b$ dos segmentos lineales. Elegimos un segmento cualquiera que permanecerá fijo - la unidad lineal -, al cual denotamos 1. Luego, sobre uno de los lados de un triángulo rectángulo trazamos desde el vértice $O$ los segmentos 1 y $b$, mientras que sobre el otro lado trazamos el segmento $a$. Seguidamente unimos el punto final del segmento 1 y el punto final del segmento a y desde el punto final del segmento $b$ trazamos la paralela a $1 a$ (Figura 4 ). Esta línea determina un segmento $c$ sobre el otro lado, al cual llamamos el producto del segmento $a$ por el segmento $b$ y designamos como $c=a b$ (cf. Hilbert, 1899, p. 33).

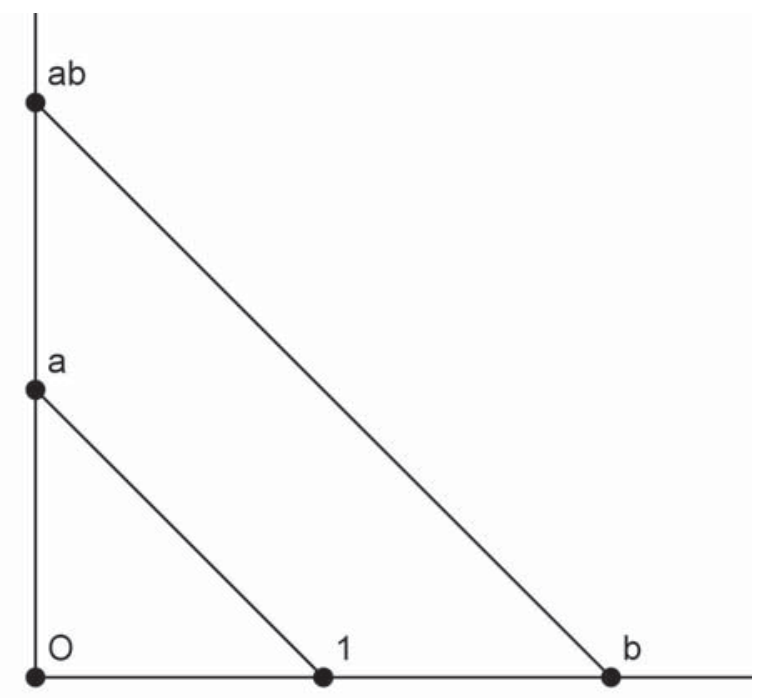

Figura 4. Producto de segmentos lineales.

Una vez definido de esta manera el producto de segmentos lineales, Hilbert prueba que cumple con todas las propiedades identificadas previamente para esta operación; en particular, el teorema de Pascal, anteriormente demostrado sin recurrir al axioma de Arquímedes, resulta esencial para probar la propiedad conmutativa del producto: $a b=b a$. La demostración procede esquemáticamente como sigue: en primer término, construimos el segmento $a b$ tal como se indicó recién. Luego trazamos el segmento $a$ sobre el primer lado del triángulo rectángulo y el segmento $b$ sobre el segundo lado. Ahora unimos el punto final de este segmento $b$ con el segmento del segmento 1 , y trazamos la paralela a $\mathrm{l} b$ que pasa por el punto $a$. Esta línea paralela determina así el segmento ba sobre el otro lado del triángulo, el cual coincide con el segmento $a b$ construido inicialmente (figura 5 ). 


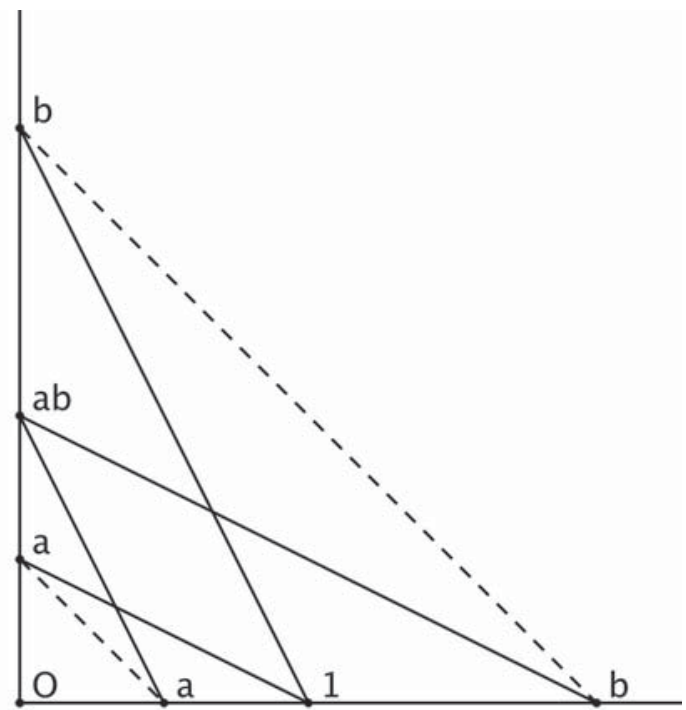

Figura 5. Conmutatividad del producto de segmentos lineales.

El aspecto central de la prueba reside en que el segmento $a b$ coincide con el segmento ba gracias al teorema de Pascal, tal como queda reflejado en el diagrama. Es decir, si unimos los puntos $a$ y $b$ sobre cada uno de los lados del triángulo recto respectivamente entre sí, obtenemos una configuración de tres pares de puntos y líneas cuyas relaciones de intersección coinciden con las descriptas en el teorema de Pascal, de acuerdo con la versión antes indicada. De este modo, el mencionado teorema le permite a Hilbert demostrar la propiedad conmutativa para el producto de segmentos lineales. A continuación, utiliza una estrategia similar para probar la ley asociativa para el producto y la ley distributiva para el producto y la suma (cf. Hilbert, 1899, p. 34-5).

Hasta aquí se ha definido una aritmética para segmentos lineales, en donde se cumplen las leyes asociativa y conmutativa para la adición, las leyes asociativa y conmutativa para el producto, y la ley distributiva para la adición y el producto. Con la ayuda de esta aritmética de segmentos es posible reconstruir la teoría de las proporciones y de los triángulos semejantes de Euclides, sin hacer uso del axioma de Arquímedes. Hilbert no se detiene a desarrollar estas teorías en detalle, sino que se limita a presentar una nueva definición de proporcionalidad y a demostrar, utilizando su aritmética de segmentos, el teorema fundamental de la teoría de las proporciones, i.e, la proposición VI.2 de los Elementos (cf. Hilbert, 1899., §16). La definición de proporcionalidad, basada en su definición previa de producto de segmentos lineales, es la siguiente (Hilbert, 1899, p. 36): 
Definición. Si $a, b, a^{\prime}, b^{\prime}$ son cuatro segmentos lineales, entonces la proporción

$$
a: b=c: d
$$

no denota sino la igualdad de segmentos lineales

$$
a b^{\prime}=a^{\prime} b
$$

Asimismo, para definir la noción de semejanza entre dos triángulos, Hilbert no recurre a la igualdad de la "razón" entre los lados correspondientes, sino en cambio a la congruencia de los ángulos de los triángulos.

Definición. Dos triángulos se llaman semejantes si sus ángulos correspondientes son congruentes (Hilbert, 1899, p. 35).

Finalmente, tras demostrar que si los segmentos $a, b, a^{\prime}, b^{\prime}$ son los lados correspondientes de dos triángulos entonces la definición anterior de proporcionalidad es válida (Teorema 22; Hilbert, 1999, teorema 411), Hilbert enuncia el teorema fundamental de la proporcionalidad, en una versión adaptada a su propia teoría:

Teorema 23: Si dos rectas paralelas determinan respectivamente, en los lados de un ángulo cualquiera, los segmentos $a, b$ y $a^{\prime}, b^{\prime}$, entonces se verifica la proporción

$$
a: b=a^{\prime}: b^{\prime}
$$

Recíprocamente, si cuatro segmentos $a, b, a^{\prime}, b^{\prime}$ satisfacen esta proporción, y $a, a^{\prime}$ y $b, b^{\prime}$ son construidos de a pares en los lados de un ángulo cualquiera, entonces las líneas que unen a los puntos finales de $a, a^{\prime}$ y $b$, b' son paralelas (Hilbert, 1899, p. 37; Hilbert, 1999, teorema 42).

Una vez enunciados eses conceptos fundamentales de su nueva teoría de las proporciones, Hilbert extiende fácilmente esta aritmética de segmentos para que incluya también relaciones de orden, de modo que se cumplan todas las propiedades de un cuerpo ordenado. Hilbert procede de la siguiente manera: en primer lugar, a la aritmética de segmentos antes definida le añadimos otro conjunto de tales segmentos. Por medio de los axiomas de orden, es fácil distinguir sobre una línea una dirección "positiva" y una “negativa”. Un segmento $A B$, denotado antes $a$, continuará llamándose $a$ si $B$ se encuentra en dirección positiva respecto de $a$; en caso contrario, se lo designará - $a$. Asimismo, un punto $A$ cualquiera se designará ahora como 0 . El segmento $A B$ es entonces positivo o mayor que 0 (en símbolos, $a>0$ ); el segmento - $a$ se designa negativo o menor que 0 (en símbolos, - $a<0$ ) (cf. Hilbert, 1899, p. 37-8). Introducidas de ese modo 
las relaciones de orden en la aritmética para segmentos, es posible probar, utilizando los axiomas I-III, la existencia de un elemento neutro y de un elemento inverso para la suma y para la multiplicación. Hilbert concluye entonces que su aritmética de segmentos lineales satisfice todas las propiedades de un cuerpo ordenado. ${ }^{34}$

Por último, para culminar con la "introducción del número", Hilbert muestra cómo que es posible introducir coordenadas en la geometría utilizando la aritmética de segmentos previamente desarrollada. Para ello procede esquemáticamente de la siguiente manera: en un plano $\alpha$ en donde se cumplen todos los axiomas I-IV (incidencia, orden, paralelas, congruencia) trazamos dos rectas perpendiculares que se intersecan en un punto 0 , las cuales nos servirán como los ejes fijos de coordenadas $X, Y$. Sobre cada una de estas rectas trazamos desde 0 los segmentos $x$, respectivamente. Seguidamente trazamos dos rectas perpendiculares a $X, Y$ desde los puntos finales de los segmentos $x, y$; la intersección de ambas rectas determinan el punto $P$. Los segmentos $x$, $y$ se llaman así las coordenadas de $P$. Y todo punto en el plano $\alpha$ está unívocamente determinado por sus coordenadas $x, y$, que pueden ser segmentos positivos, negativos o 0 .

De este modo, los resultados de la teoría de las proporciones anteriormente desarrollada nos proporcionan fácilmente la ecuación de la recta. Sea $l$ una recta cualquiera sobre el plano $\alpha$ que pasa por 0 y por un punto $C$, cuyas coordenadas son el par ordenado $(a, b)$. Si $x, y$ son las coordenadas de un punto cualquiera de $l$, entonces por el teorema 23 se cumple que $a: b=x: y$. Dada la definición de proporcionalidad enunciada por Hilbert, ello es lo mismo que decir que $b x-a y=0$, o sea, la ecuación (general) de la recta (ver fig. 6).

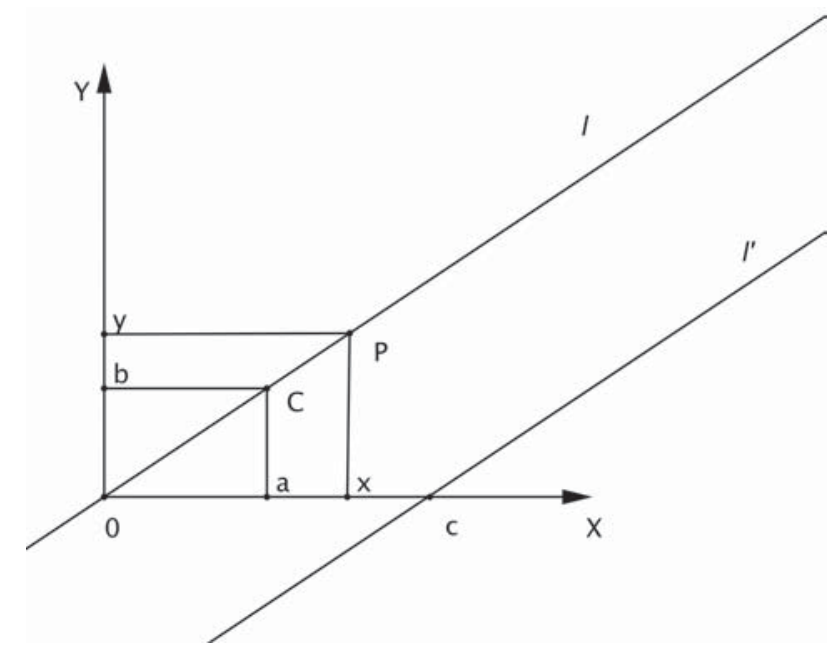

Figura 6. Introducción de un sistema de coordenadas (cartesianas).

34 "En esta aritmética de segmentos todas las reglas de las operaciones 1-16, enumeradas en la sección 13, son válidas" (Hilbert, 1899, p. 37). 
Obtenida de este modo la ecuación general de la recta, Hilbert da por culminada su exposición en torno a cómo es posible introducir un sistema de coordenadas en la geometría "desde dentro", es decir, de un modo puramente geométrico, que no recurre a un cuerpo numérico en particular como una imposición desde fuera. La conclusión que extrae del procedimiento que hemos analizados es la siguiente:

A partir de estos desarrollos concluimos, de un modo independiente al axioma de Arquímedes, que toda línea en el plano puede ser representada por medio de ecuaciones lineales a través de las coordenadas $x, y$, e inversamente, que todas las ecuaciones lineales de tal clase, en la que los coeficientes son segmentos en la geometría dada, representan una línea. (...) La construcción subsiguiente de la geometría puede ser realizada por medio de los métodos habituales que son utilizados en la geometría analítica (Hilbert, 1899, p. 3).

La técnica desarrollada por Hilbert permite probar que, dados un plano $\alpha$ que satisface los axiomas I-IV y un cuerpo ordenado $\mathbb{K}$ asociado a la aritmética de segmentos en $\alpha$, el plano $\alpha$ es isomorfo a un plano cartesiano $\mathbb{K}^{2}$ sobre el cuerpo $\mathbb{K}$. Asimismo, si junto con los axiomas I-IV se asume además el axioma de Arquímedes, entonces es posible asignar un número real a cada punto sobre la línea (cf. Hilbert, 1899, p. 38-9). ${ }^{3} 5$ Por último, esta construcción de una aritmética para segmentos lineales exhibe la conexión fundamental entre dos teoremas clásicos de la geometría proyectiva, como los teoremas de Desargues y Pappus (Pascal), y las propiedades algebraicas de las operaciones definidas geométricamente para los segmentos lineales. Más precisamente, Hilbert muestra que mientras que el primero asegura que el plano puede ser coordenatizado por medio de un cuerpo no conmutativo (siendo esencial para probar la propiedad asociativa de la multiplicación), el segundo garantiza la propiedad conmutativa para la misma operación (cf. Hilbert, 1899, cap. 5).

\section{Consideraciones FinALES: EL MOdERNo MÉTodo AXIOMÁTico Y LA UNIDAD DE LA MATEMÁTICA}

Para concluir quisiera realizar algunas observaciones generales respecto del significado de la aritmética de segmentos lineales presentada en Fundamentos de la geometría (1899). En primer lugar, las investigaciones de Hilbert contribuyeron notablemente a

$3_{5}$ En la primera edición francesa de Fundamentos de la geometría (Hilbert, 1900c), Hilbert incorpora su novedoso axioma de completitud, que permite garantizar una correspondencia uno-a-uno con los números reales. 
esclarecer un problema de enorme importancia para los fundamentos de la geometría, a saber: la determinación del papel que el número desempeña en la geometría, y en particular, la función de los axiomas de continuidad en la introducción de coordenadas numéricas. Como hemos visto, sus reflexiones se originaron en gran medida en las dificultades planteadas por los métodos geométricos puros introducidos von Staudt en la geometría proyectiva en la mitad del siglo xıx, y estuvieron motivadas no sólo por la búsqueda de una solución a estos problemas matemáticos concretos, sino también por preocupaciones de carácter epistemológico y metodológico. El procedimiento pensado por Hilbert para introducir coordenadas en la geometría euclídea, basado en su novedoso cálculo de segmentos, reveló que efectivamente era posible introducir un sistema de coordenadas de un modo puramente geométrico y sin utilizar ningún axioma de continuidad, en particular, el axioma de Arquímedes. Más aún, estos resultados exhibieron por primera vez la potencialidad de los teoremas de Desargues y Pascal para realizar una coordenatización interna de la geometría elemental.

Cabe aclarar, sin embargo, que de ningún modo estos resultados constituyeron la culminación de estos problemas, sino que más bien fueron el punto de partida para nuevas investigaciones. Por ejemplo, poco después Hessenberg (1905b) mostró cómo era posible construir un cálculo de puntos geométricos similar a la aritmética de segmentos hilbertiana, obteniendo de ese modo una simplificación de su aritmetización interna de la geometría. Asimismo, algunas de las ideas originales de Hilbert fueron posteriormente mejoradas. Especialmente, la teoría de las proporciones que, según Freudenthal (1957, p. 127), aparece como "complicada y oscura" desde un punto de vista más contemporáneo, fue simplificada notablemente por Bernays (1999), en un trabajo que fue publicado como suplemento en la décima edición de Fundamentos de la geometría (Hilbert, 1999). Los resultados de Hilbert motivaron así un gran número de fructíferas investigaciones (cf. Karzel \& Kroll, 1988; Pambuccian, 2013).

En segundo lugar, la aritmética para segmentos lineales permitió "sortear el hiato" o "trazar un puente", por así decirlo, entre las geometrías sintéticas y las geometrías analíticas. Según lo advierte el propio Hilbert, sus investigaciones revelaron cómo "es posible construir un cálculo con segmentos o una geometría analítica, en donde las letras representan de hecho segmentos, y no números" (Hilbert, 2004 [1898a], p. 261). Hilbert reconoce entonces que los segmentos lineales pueden conformar, una vez que las operaciones de adición y producto han sido definidas adecuadamente, la base de un cuerpo ordenado que puede ser a su vez utilizado para construir un sistema de coordenadas. Empero ello equivale a afirmar que, en gran medida, la geometría analítica es posible sin la imposición de cuerpos numéricos “desde afuera”. Precisamente aquí se aprecia la importancia que para Hilbert tenía el hecho de que su aritmetización de la geometría fue realizada con independencia del axioma de Arquímedes, en tanto que 
éste era el único axioma que podía considerarse como no puramente geométrico, puesto que su formulación misma suponía el concepto de número entero positivo.

Los resultados de Hilbert constituyen así una explicación de cómo y por qué existe una completa correspondencia entre la geometría sintética y la geometría analítica. Al probar que la teoría de las magnitudes surge intrínsecamente en la geometría sintética, y por lo tanto no debe ser impuesta desde fuera por medio de supuestos (numéricos) adicionales, Hilbert consigue mostrar al mismo tiempo que la suposición general que guía a la geometría analítica, o sea, la coordenatización de los puntos de una línea con los números reales, está realmente justificada. Por medio de su cálculo para segmentos lineales Hilbert brinda un fundamento axiomático para las conexiones estructurales entre la geometría euclídea la geometría analítica, una preocupación que como vimos está presente ya en sus primeros trabajos consagrados a los fundamentos de la geometría.

Finalmente, en virtud de nuestro examen, podemos comprender ahora la afirmación de Hilbert según la cual, gracias a su análisis axiomático, llegamos a "proporcionar un nuevo fundamento para la unidad de la matemática" (Hilbert, 2004 [1898b], p. 223). Su nuevo método axiomático formal le permitió mostrar cómo distintas teorías matemáticas como la geometría y la aritmética (y el análisis), que en esta etapa temprana él consideraba muy distantes respecto de sus bases epistemológicas, están conectadas estructuralmente. La contribución del método axiomático (formal) en la consecución de esta tarea se manifestó así a menos en dos puntos principales. En primer lugar, para mostrar que los segmentos lineales comparten con los números reales la estructura de un cuerpo ordenado, fue necesario contar con una axiomatización precisa de esta estructura algebraica, a partir de la cual es posible mostrar qué propiedades son compartidas por el cuerpo formado por segmentos y por el cuerpo formado por números, y cuáles no. En el Festschrift Hilbert presenta entonces el primer sistema de axiomas para un cuerpo ordenado (arquimediano). En segundo lugar, la presentación de cada una de estas estructuras como un sistema de axiomas formales es lo que hace posible, por un lado, identificar y descubrir las semejanzas estructurales; por otro lado, es lo que permite determinar qué axiomas o teoremas son responsables de cada una de las propiedades.

En resumen, además del interés y la relevancia que recaen en estos resultados desde un punto de vista estrictamente matemático, la aritmética de segmentos lineales constituye un claro ejemplo de una creencia general de Hilbert respecto de la naturaleza de la matemática y del valor del método axiomático para el conocimiento matemático. Me refiero a su conocida tesis de la "unidad de la matemática”, expresada en su conferencia de París, intitulada "Problemas matemáticos": 
En mi opinión, la matemática es un todo indivisible, un organismo cuya vitalidad está condicionada por la conexión entre sus partes. Puesto que a pesar de la variedad del conocimiento matemático, todavía somos muy conscientes de las ideas de la matemática como un todo y de las numerosas analogías en sus distintos campos de conocimiento (Wissensgebieten). También llegamos a percibir que, cuanto más avanzada o desarrollada se encuentra una teoría, más armoniosa y uniformemente procede su construcción, y relaciones insospechadas entre ramas hasta el momento separadas de las ciencias son reveladas. De este modo ocurre que a través de su extensión, el carácter orgánico de la matemática no se pierde sino que se manifiesta a sí mismo más claramente (...) La unidad orgánica de la matemática es inherente a la naturaleza de esta ciencia, porque la matemática es el fundamento de todo conocimiento exacto de los fenómenos naturales (Hilbert, 190ob, p. 329).

La aritmética de segmentos era así para Hilbert un caso concreto en donde podía percibirse este tipo de "unidad orgánica" de la matemática. Es decir, era un claro ejemplo de cómo dos disciplinas como la geometría y la aritmética y el álgebra, en apariencia muy distintas o separadas, estaban conectadas estructuralmente. En la misma conferencia de París, Hilbert destaca además que otras analogías entre el pensamiento geométrico y el pensamiento aritmético fueron reveladas por Minkowski en un trabajo de 1896 sobre la Geometría de los números (cf. Hilbert, 190ob, p. 296; Smadja, 2012). Y según hemos podido observar en sus notas de clases, Hilbert reconoció que ésta era precisamente una de las características más atractivas y fructíferas de su nuevo método axiomático, a saber: la capacidad de descubrir y exhibir conexiones hasta el momento desconocidas entre distintas teorías matemáticas, y de esa manera contribuir a la unidad del conocimiento matemático.@

Agradecimientos. Este trabajo fue realizado durante una estancia de investigación en el Max Planck Institute for the History of Science (Berlin). Agradezco a Vincenzo De Risi y a los participantes del grupo de investigación "Modern Geometry and the Concept of Space”, por sus comentarios y sugerencias. Asimismo, quisiera agradecer a Leo Corry y a los dos árbitros anónimos de Scientiae Studia, por sus observaciones a una versión preliminar de este artículo.

\section{Eduardo Nicolás Giovannini}

Consejo Nacional de Investigaciones Científicas y Técnicas,

Santa Fe, Argentina. engiovannini@conicet.gov.ar

Arithmetizing the geometry from inside: David Hilbert's segment calculus 


\begin{abstract}
On the basis of a set of unpublished notes for lecture courses on geometry, the paper seeks to contextualize and analyze one of the most important and original contributions of David Hilbert's celebrated monograph Foundations of Geometry (1899), namely its arithmetic of line segments (Streckenrechnungen). It is argued that Hilbert attributed to his arithmetic of segments an important epistemological and methodological meaning, in addition to its relevance as an original mathematical result. In particular, it is claimed that for Hilbert his arithmetic of segments represented a clear example of one of the most fruitful and attractive traits of his new formal axiomatic method, i.e., the power to discover and exhibit inner or structural connections among different mathematical theories.
\end{abstract}

KEYWORDS • Hilbert. Axiomatic method. Unity of mathematics. Euclidean geometry. Arithmetization.

\title{
REFERENGIAS BIBLIOGRÁFIGAS
}

Arana, A. \& Mancosu, P. On the relationship between plane and solid geometry. The Review of Symbolic Logic, 5, 2, p. 294, -353 , 2012.

Bernays, P. Vereinfachte Begründung der Proportionlehre. In: Toepeld, M. (Ed.). Hilbert Grundlagen der Geometrie. Stuttgart/Leipzig: Teubner, 1999. Anexo 2, p. 243-8.

Blumenthal, O. David Hilbert. Die Naturwissenschaften, 4, p. 67-72, 1922. . Lebensgeschichte. In: Hilbert, D. Gesammelte Abhandlungen. Berlin: Springer, 1935. v. 3, p. 388429 .

Bos, H. On the representation of curves in Descartes' Géométrie. Archive for History of Exact Sciences, 24, 4, p. 295-338, 1981 .

Cantor, G. Über die Ausdehnung eines Satzes aus der Theorie der trigonometrischen Reihe. Mathematische Annalen, 5, p. 123-32, 1872 .

Contro, W. Von Pasch bis Hilbert. Archives for History of Exact Sciences, 15, p. 283-95, 1976.

Corry, L. David Hilbert and the axiomatization of physics, 1898-1918: from 'Grundlagen der Geometrie' to 'Grundlagen der Physik'. Dordrecht: Kluwer, 2004.

Darboux, G. Sur le théorème fundamental de la géométrie projective. Mathematische Annalen, 17, p. 5561,1880 .

Epple, M. Styles of argumentation in late 19th century geometry and the structure of mathematical modernity. In: Отте, M. \& PAnZA, M. (Ed.). Analysis and synthesis in mathematics. History and philosophy. Dordrecht: Kluwer, 1997. p. 177-98.

Euclides. Elementos. Traducción M. L. P. Castaños. Madrid: Gredos, 1994.

FerReirós, J. O $\theta \varepsilon o \zeta \alpha ́ \alpha p \theta \mu \eta \tau i \zeta \varepsilon l:$ the rise of pure mathematics as arithmetic with Gauss. In: Goldstein, C.; Schappacher, N. \& Schwermer, J. (Ed.). The shaping of arithmetic: number theory after Carl Friedrich Gauss's Disquistiones Arithmeticae. Berlin: Springer Verlag, 2006. p. 235-68.

Flament, D. \& Nabonnand, P. (Ed.). Justifier en mathématiques. Paris: Éditions de la Maison des Sciences de L'Homme, 2011.

Folkerts, M. (Ed.). Mathemata. Festschrift für Helmuth Gericke. Stuttgart: Franz Steiner Verlag, 1985.

Freudenthal, H. Zur Geschichte der Grundlagen der Geometrie. Zugleich einer Besprechung der 8. Auflage von Hilberts "Grundlagen der Geometrie". Nieuw Archief voor Wiskunde, 4, p. 105־42, 1957.

Gauss, C. \& Bessel, F. Briefwechsel. Herausgegeben aufderköniglich Preussischen Akademie der Wissenschaften. Leipzig: Engelman, 1880. 
Giovannini, E. Completitud y continuidad en "Fundamentos de la geometría" de Hilbert: acerca del Vollständigkeitsaxiom. Theoria, 28, 76, p. 139-63, 2013.

Goldstein, G.; Schappacher, N. \& Schwermer, J. (Ed.). The shaping of arithmetic: number theory after Carl Friedrich Gauss's Disquistiones Arithmeticae. Berlin: Springer Verlag, 2006.

Grattan-Guinness, I. Numbers, magnitudes, ratios, and proportions in Euclid's Elements: how did he handle them? Historia Mathematica, 23, p. 355-75, 1996.

GraY, J. Worlds out of nothing: a course in the history of geometry in the 19 th century. London: Springer, 2007. Greenberg, M. J. Euclidean and non-euclidean geometries. 3. ed. New York: Freeman, 2000.

Hallett, M. Reflections on the purity of method in Hilbert's Grundlagen der Geometrie. In: Mancosu, P. (Ed.). The philosophy of mathematical practice. New York: Oxford University Press, 2008. p. 198-255.

Hartshorne, R. Geometry: Euclid and beyond. New York: Springer, 2000.

Heath, T. L. The thirteen books of Euclid's Elements. 2 ed. New York: Dover Publications, 1956. 3v.

Hendriks, V.; Pedersen, S. \& Jørgensen, K. (Ed.). Proof theory. History and philosophical significance. Dordrecht: Kluwer, 2000.

Hessenberg, G. Beweis des Desargues'schen Satzes aus dem Pascal. Mathematische Annalen, 61, p. 16172,1905 a.

. Über einen geometrischen Calcül. Acta Mathematica, 29, p. 1-24, 1905b.

Hilbert, D. Grundlagen der Geometrie. In: Herausgegeben von dem Fest-Comitee. Festschrift zur Feier der Enthüllung des Gauss-Weber-Denkmals in Göttingen . 1. ed. Leipzig: Teubner, 1899.

Über den Zahlbegriff. Jahresbericht der Deutschen Mathematiker, 8, p. 180-4, 1900a.

. Mathematische Probleme. Vortrag, gehalten auf dem internationalen Mathematiker-Kongreß zu Paris 1900. Nachrichten von der königl. Gesellschaft der Wissenschaften zu Göttingen. Mathematischphysikalische Klasse, 1900b. p. 253-97.

. Gesammelte Abhandlungen. Berlin: Springer, 1935 .

Über die gerade Linie als kürzeste Verbindung zweier Punkte. Aus einem an Herrn F. Klein gerichteten Briefe. In: ToepeLL, M. (Ed.). Hilbert Grundlagen der Geometrie. Stuttgart/Leipzig: Teubner, 1999 [1895]. Anexo 1, p. 126-32.

. Projektive Geometrie (cod. ms. D. Hilbert 535, 1891). In: Majer, U. \& Hallett, M. (Ed.). David Hilbert's lectures on the foundations of geometry, 1891-1902. Berlin: Springer, 2004 [1891]. p. 21-55.

. Die Grundlagen der Geometrie (cod. ms. D. Hilbert 541, 1893/4). In: Majer, U. \& Hallett, M. (Ed.). David Hilbert's lectures on the foundations of geometry, 1891-1902. Berlin: Springer, 2004, [1893/1894]. p. $72-178$.

. Grundlagen der Euklidischen Geometrie (cod. ms. D. Hilbert 541, 1898). In: Majer, U. \& Hallett, M. (Ed.). David Hilbert's lectures on the foundations of geometry, 1891-1902. Berlin: Springer, 2004, [1898a]. p. 221-86.

. Elemente der Euklidischen Geometrie (cod.ms. D. Hilbert 552, 1898). In: Majer, U. \& Hallett, M. (Ed.).

David Hilbert's lectures on the foundations of geometry, 1891-1902. Berlin: Springer, 2004, [1898b]. p. 3०4, -402 .

. Grundlagen der Geometrie (ms. Vorlesung, SS 1902). In: Majer, U. \& Hallett, M. (Ed.). David Hilbert's lectures on the foundations of geometry, 1891-1902. Berlin: Springer, 2004 [1902]. p. 540-606.

. Wissenchaftliche Tagebücher. Niedersächsische Staats und Universitätsbibliothek Göttingen, Handschriftenabteilung, Cod. Ms. D. Hilbert 600, s/f.

HöLdER, O. Streckenrechnung und projective Geometrie. Berichte über die Verhandlungen der königlich sächsischen Gesellschaft der Wissenschaften zu Leipzig, Mathematisch-physische Klasse, 63, 2, p. 65-183, 1911. 
Karzed, H. \& Krold, H. J. Geschichte der Geometrie seit Hilbert. Darmstadt: Wissenschaftliche Buchgesellschaft, 1988.

KLEIN, F. Über die sogenannte Nicht-Euklidische Geometrie (zweiter Aufsatz). Mathematische Annalen, 6, p. $112-45,1873$.

. Nachtrag zu dem “zweiten Aufsatz über Nicht-Euklidische Geometrie”. Mathematische Annalen, 7 , p. $5^{31-7}, 1874$.

. Elementary mathematics from an advanced point of view. New York: Dover Publications, 1949. v. 1: Geometry.

Kuine, M. El pensamiento matemático de la antiguëdad a nuestros días. Madrid: Alianza Editorial, 1992. v. 3.

Majer, U. \& Hallett, M. (Ed.). David Hilbert's lectures on the foundations of geometry, 1891-1902. Berlin: Springer, 2004.

Mancosu, P. (Ed.). The philosophy of mathematical practice. New York: Oxford University Press, 2008.

Minkowsкi, H. Geometrie der Zahlen. Leipzig: Teubner, 1896.

Mueller, I. Philosophy of mathematics and deductive structure in Euclid's Elements. Massachusetts: The MIT Press, 1981.

Nabonnand, P. La théorie des Würfe de von Staudt: une irruption de l'algèbre dans la géométrie pure. Archives for History of Exact Sciences, 62, p. 201-42, 2008a.

. Contributions à l'histoire de la géométrie projective au xIxe siècle. Document presenté pour l' HDR. 2006. Disponible en: 〈https://hal.archives-ouvertes.fr/hal-o1082207/document>. Acceso en 20 ene. 2015 .

. L' argument de la généralité chez Carnot, Poncelet et Chasles. In: Flament, D. \& Nabonnand, P. (Ed.). Justifier en mathématiques. Paris: Éditions de la Maison des Sciences de L'Homme, 2011. p. 17-47.

NAGEL, E. The formation of modern conceptions of logic in the development of geometry. Osiris, $7, \mathrm{p}$. $14,2-223,1939$.

Отте, M. \& Panza, M. (Ed.). Analysis and synthesis in mathematics. History and philosophy. Dordrecht: Kluwer, 1997 .

Pambuccian, V. Review of David Hilbert's lectures on the "Foundations of geometry", 1891-1902. Philosophia Mathematica, 21, 2, p. 255-75, 2013.

Pasch, M. Vorlesungen überneuere Geometrie. 2. ed. Leipzig: Teubner, 1926 [1882].

REYE, T. Geometrie der Lage. Leipzig: Baumgärtner, 1886.

ReID, C. Hilbert. New York: Springer, 1996.

Rowe, D. The calm before the storm: Hilbert's early views on foundations. In: Hendriks, V.; Pedersen, S. \& Jørgensen, K. (Ed.). Proof theory. History and philosophical significance. Dordrecht: Kluwer, 2000. p. $55^{-9} 3$.

Schlimm, D. Pasch's philosophy of mathematics. The Review of Symbolic Logic, 3, 1, p. 1-26, 2010.

Schur, F. Über den Fundamentalsatz der projectiven Geometrie. Mathematische Annalen, 51, p. 4,01-9, 1898.

Smadja, I. Local axioms in disguise: Hilbert on Minkowski diagrams. Synthese, 186, p. 315-70, 2012.

Toepeld, M. Zur Schlüsselrolle Friedrich Schurs bei der Entstehung von David Hilberts "Grundlagen der Geometrie”. In: Folkerts, M. (Ed.), Mathemata. Festschrift für Helmuth Gericke. Stuttgart: Franz Steiner Verlag, 1985 . p. $637-49$.

Über die Entstehung von David Hilberts Grundlagen der Geometrie. Göttingen: Vandenhoeck/ Ruprecht, 1986.

Toepeld, M. (Ed.). Hilbert Grundlagen der Geometrie. Stuttgart/Leipzig: Teubner, 1999.

Voelke, J. D. Le theoreme fondamental de la géométrie projective: évolution de sa preuve entre 1847 et 1900. Archives for History of Exact Sciences, 62, p. 24,3-96, 2008. 
Von Staudt, G. Geometrie der Lage. Nürnberg: Verlag von Bauer und Raspe, 1847 .

Wевв, J. Mechanism, mentalism, and metamathematics. An essay on finitism. Dordrecht: Reidel, 1980.

Wiener, H. Über Grundlagen und Aufbau der Geometrie. Jahresbericht der Deutschen MathematikernVereinigung, 1, p. 45-8, 1891 .

. Weiteres über Grundlagen und Aufbau der Geometrie. Jahresbericht der Deutschen MathematikernVereinigung, 3, p. 70-8०, 1893. 